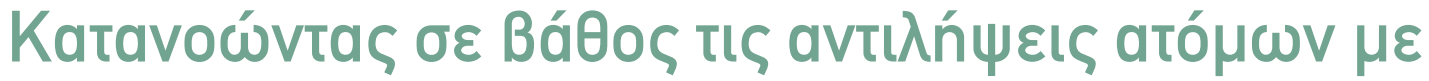

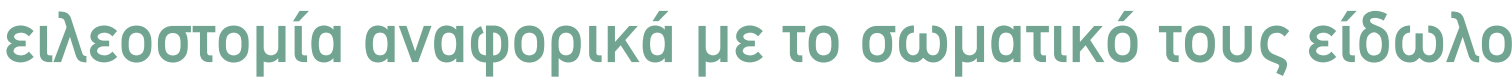

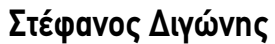

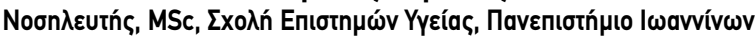

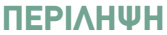

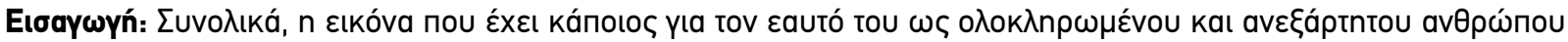

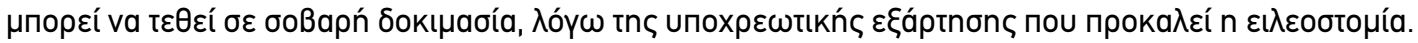

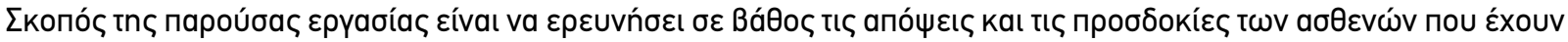

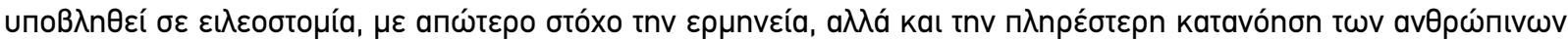

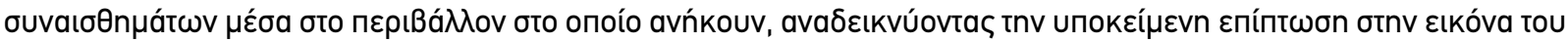
Eautoú tous.

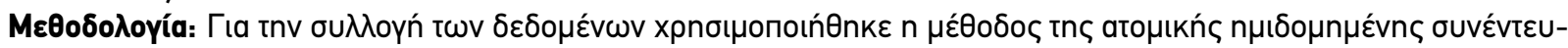

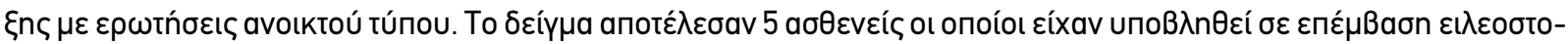

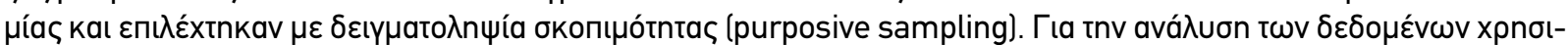

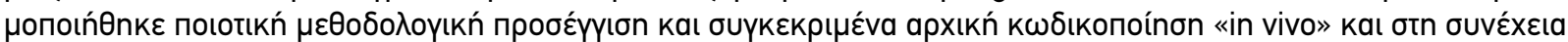
$\theta \varepsilon \mu a t ı k n ́ ~ a v a ́ \lambda u \sigma n ~ n \varepsilon p ı \varepsilon x o \mu \varepsilon ́ v o u$ (content analysis).

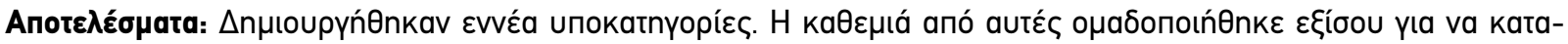

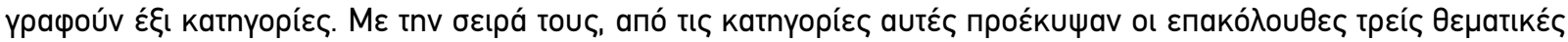

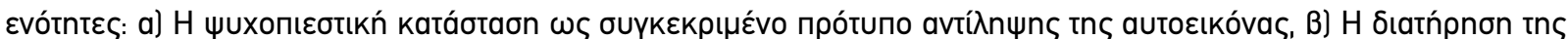

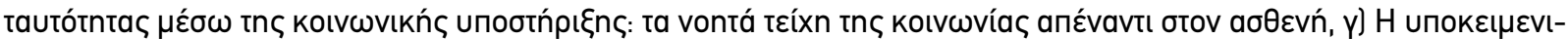

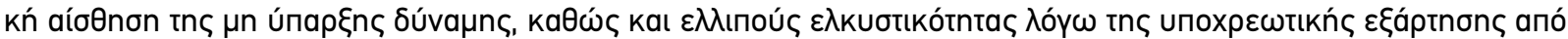

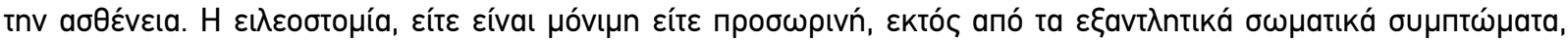

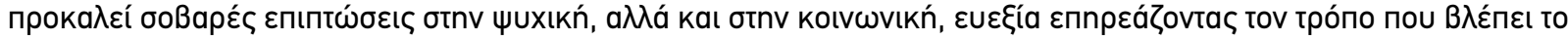

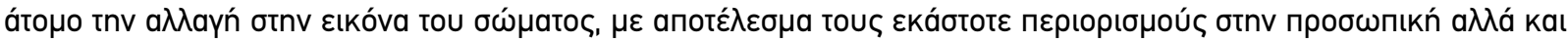

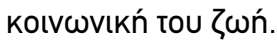

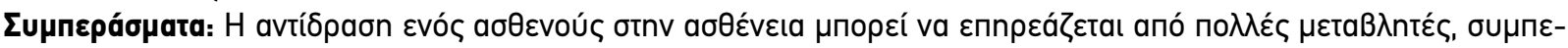

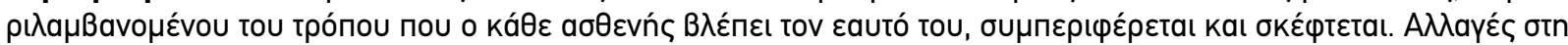

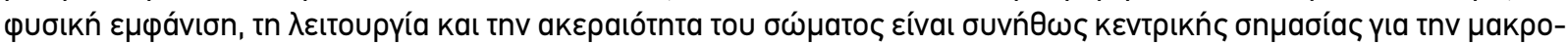

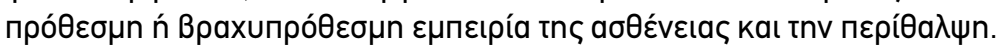

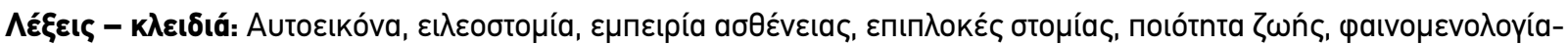
$\varepsilon \rho \mu n v \varepsilon U t i k n ́$. 


\section{BAEIKA EHMEIA}

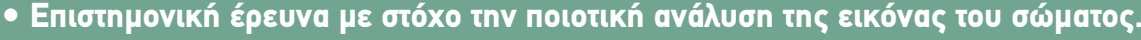

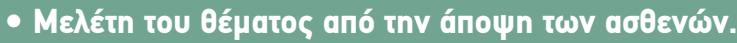

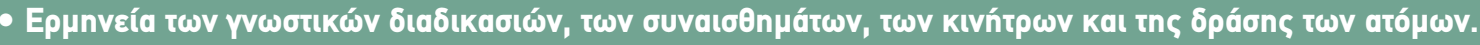

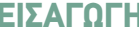

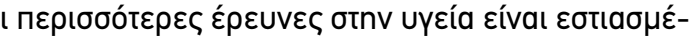

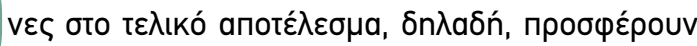

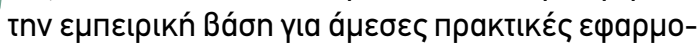

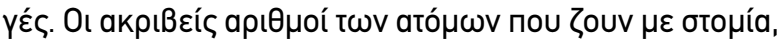

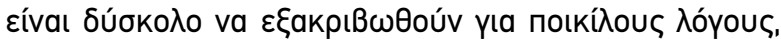

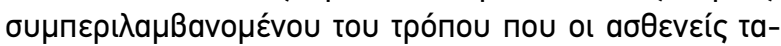

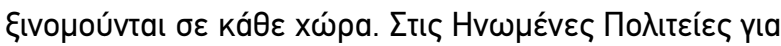

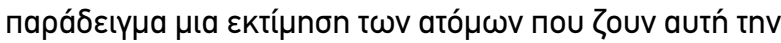

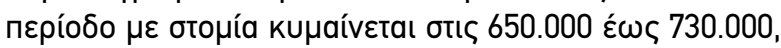

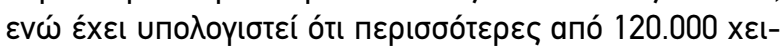

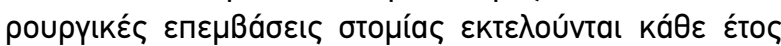
(WOCN 2016).

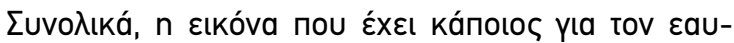

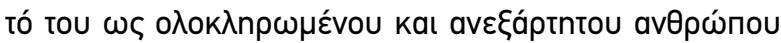

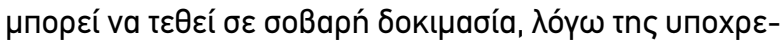

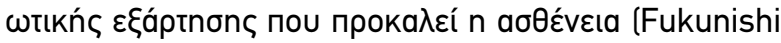

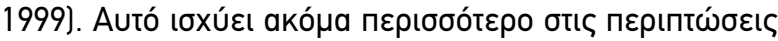

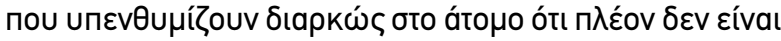

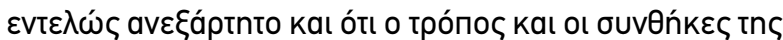

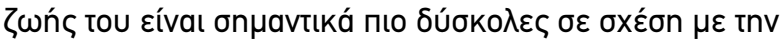

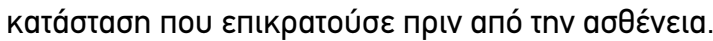

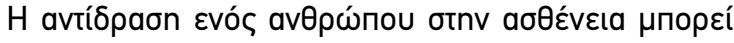

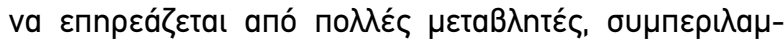

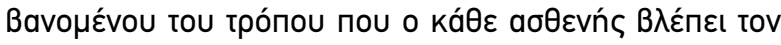

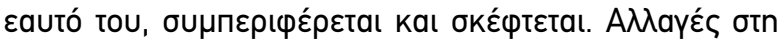

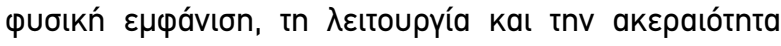

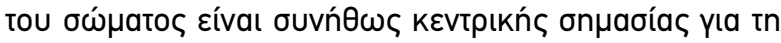

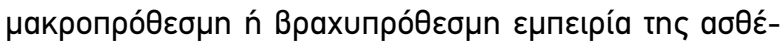

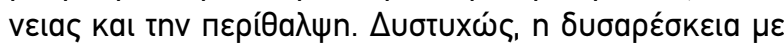

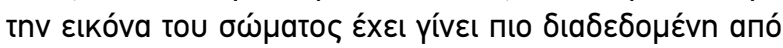

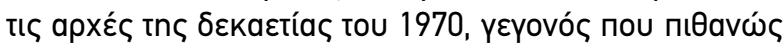
o

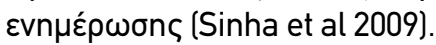

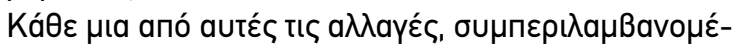

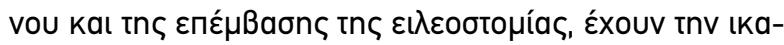

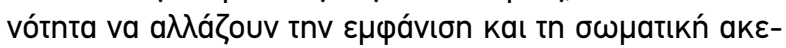

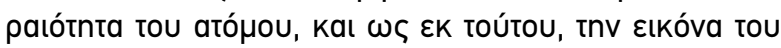

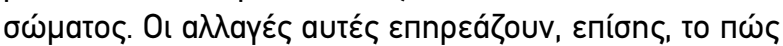

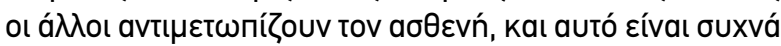

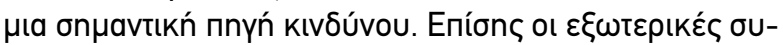

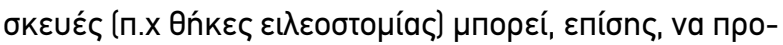

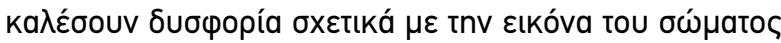

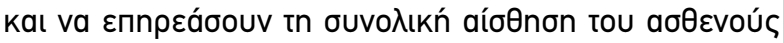

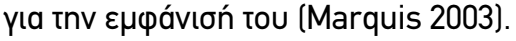

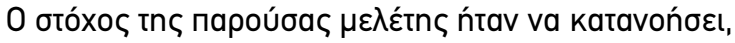

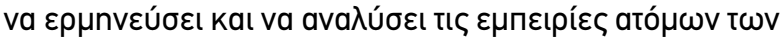

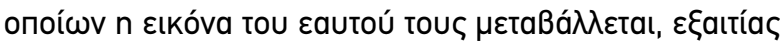

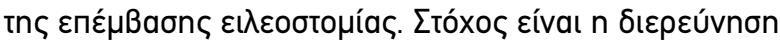

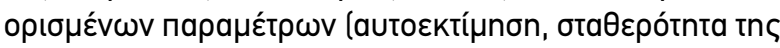

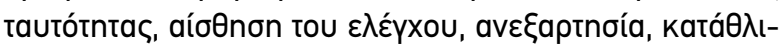

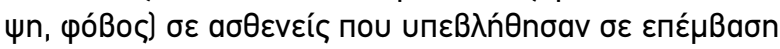

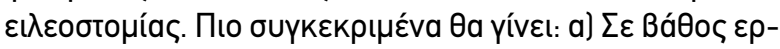

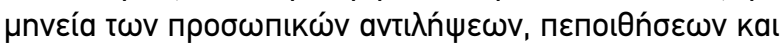

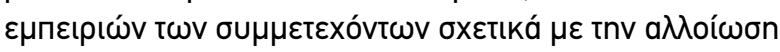

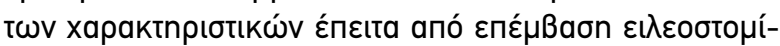

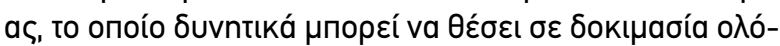

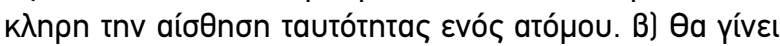

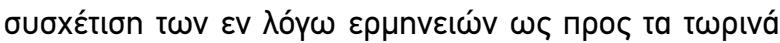

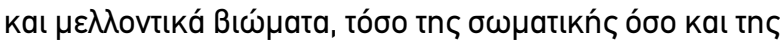

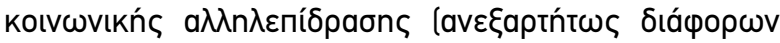

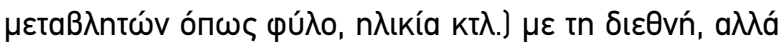

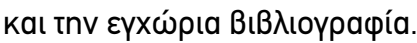

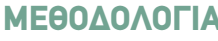

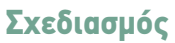

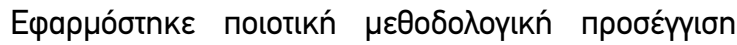

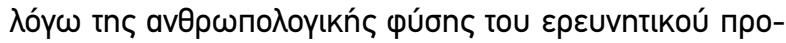

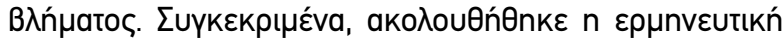

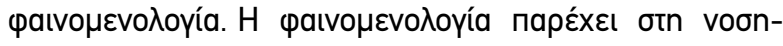

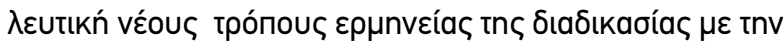

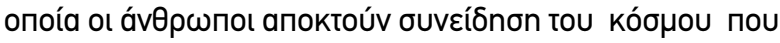

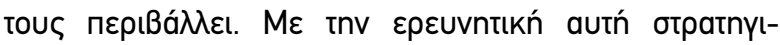

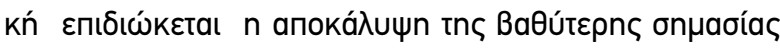

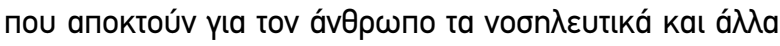

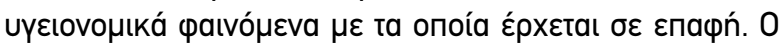

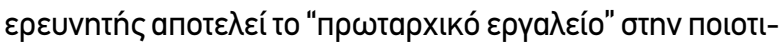

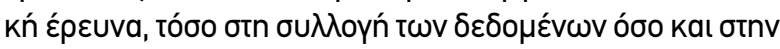

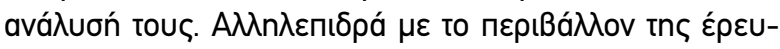

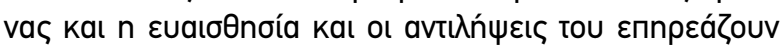

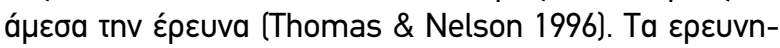

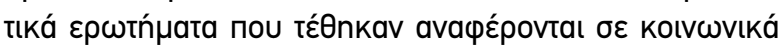

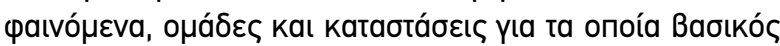

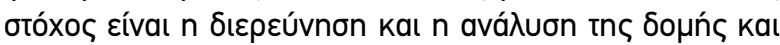

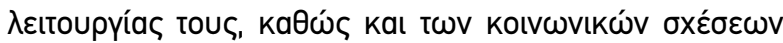

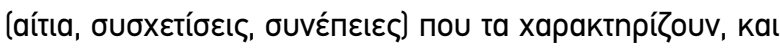

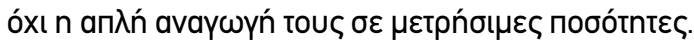




\section{EPEYNHTIKA APOPA}

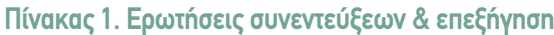

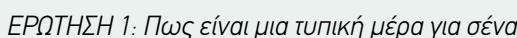

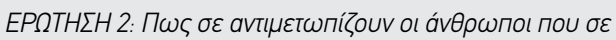

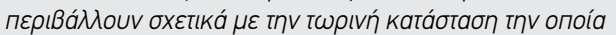

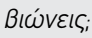

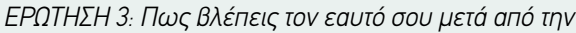

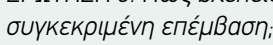

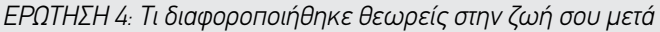

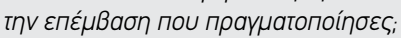

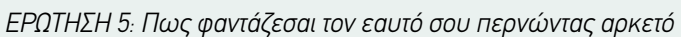

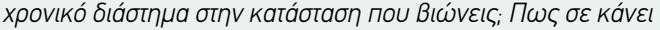
va aıఠӨáveбal:

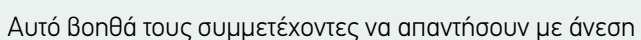

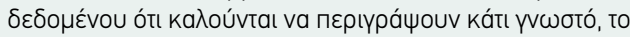

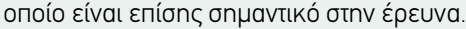

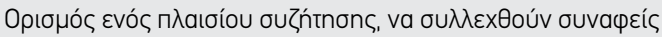

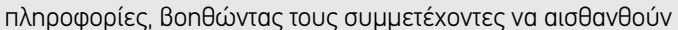

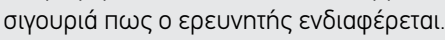

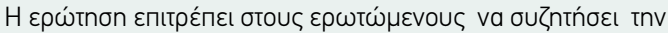
$\varepsilon \mu п \varepsilon เ \rho i ́ a$ tou/tnc

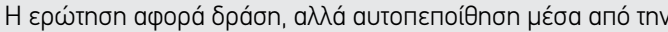

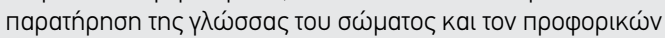

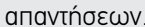

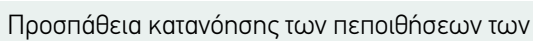

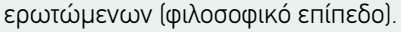

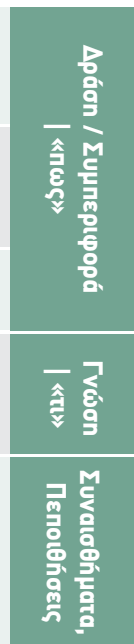

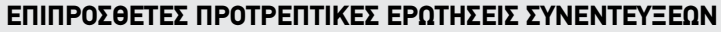

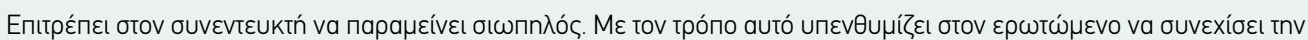
ıotopía tou.

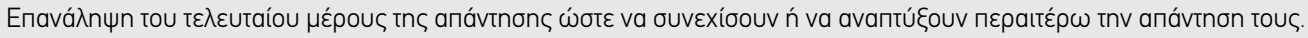

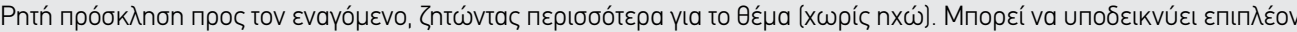

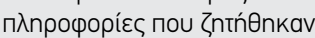

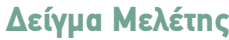

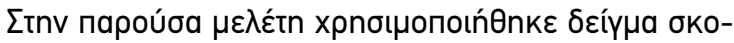

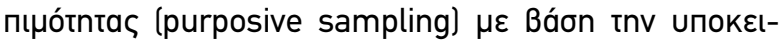

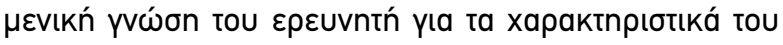

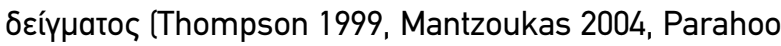

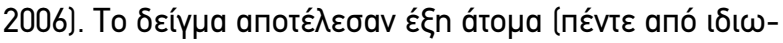

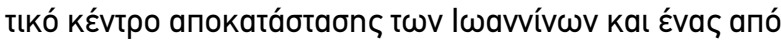

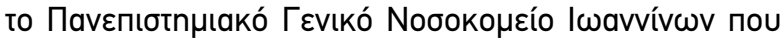

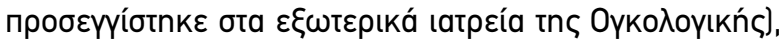

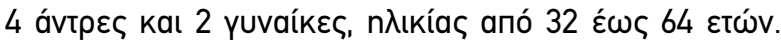

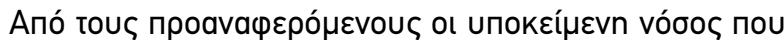

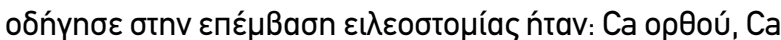

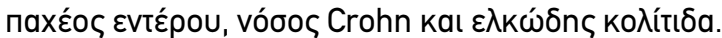

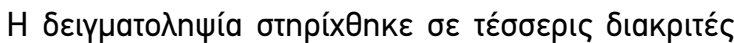

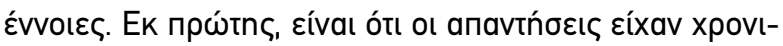

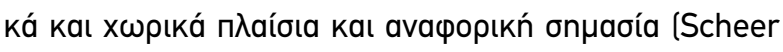

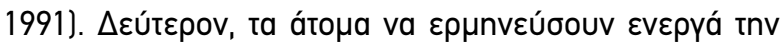

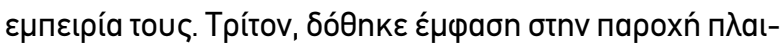

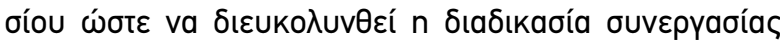

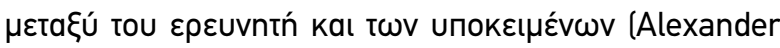

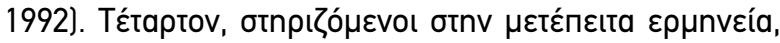

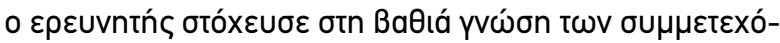

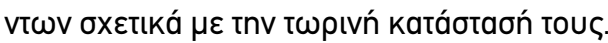

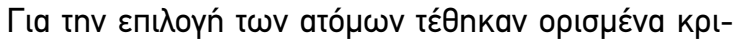

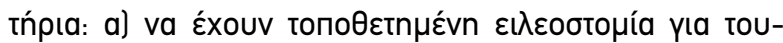

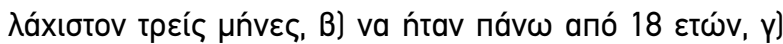

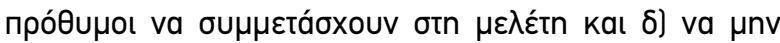

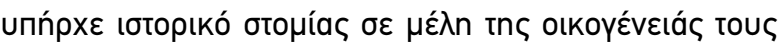

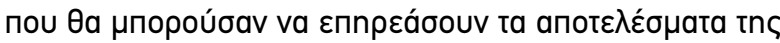

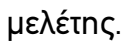




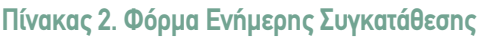

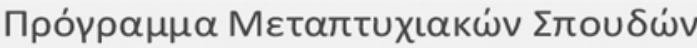

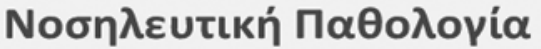

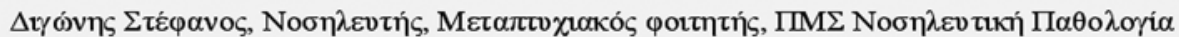
ФOPEA $\Sigma$ : ПANEIII $\Sigma$ THMIO I $A A N N I N \Omega N$ - TEI HIIEIPOY

TH人.: 6987388430

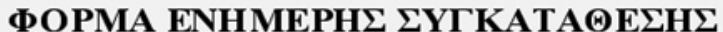

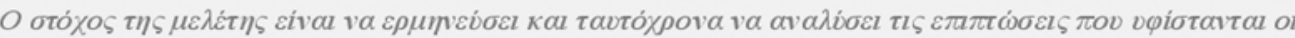

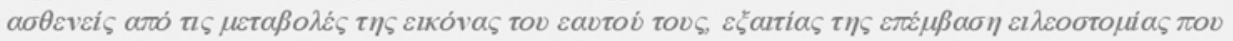

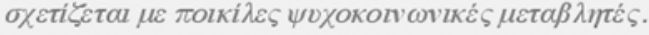

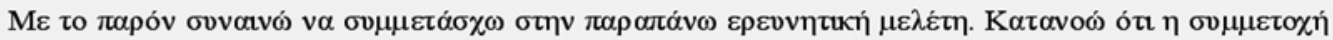

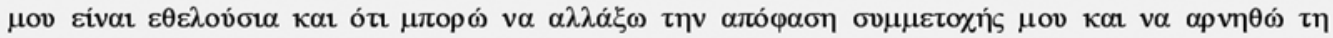

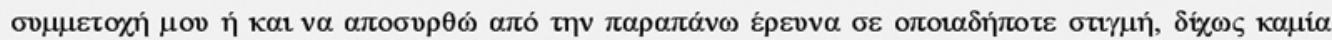

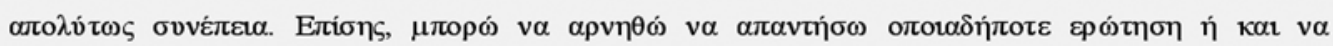

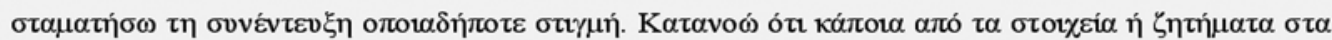

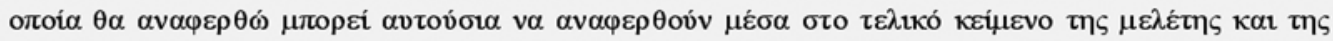

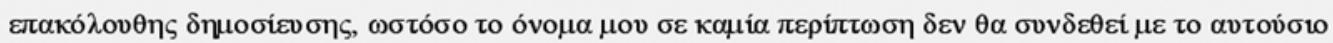

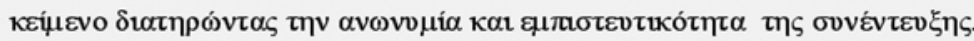

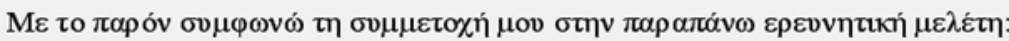
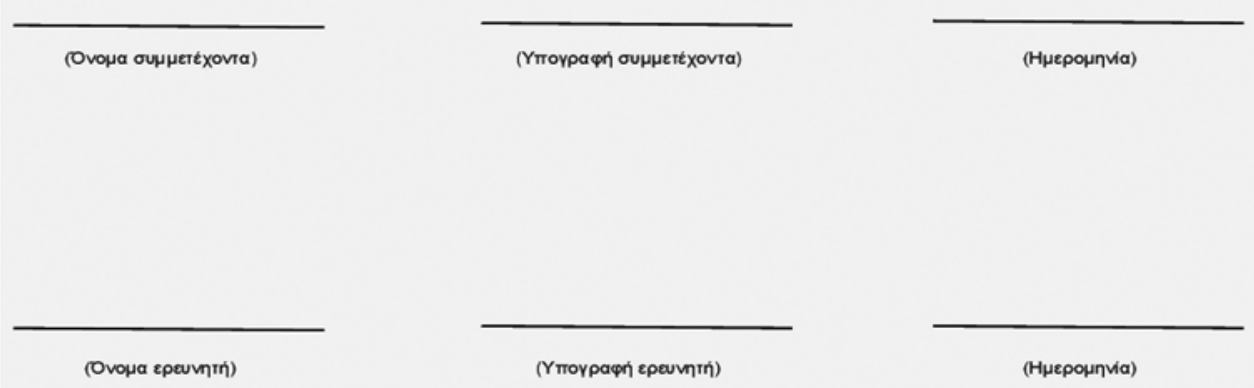

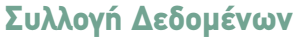

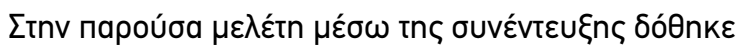

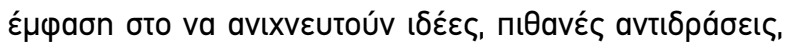

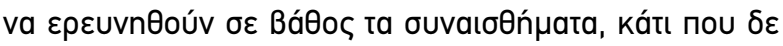

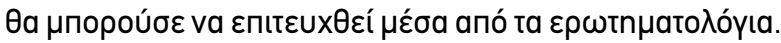

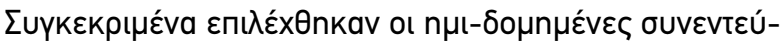

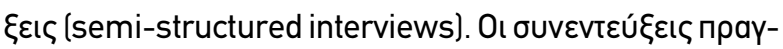

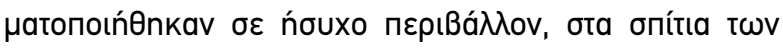

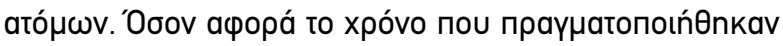

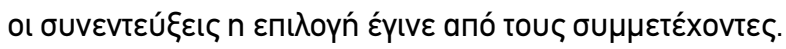




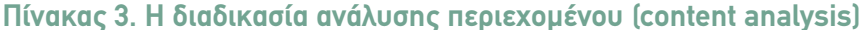

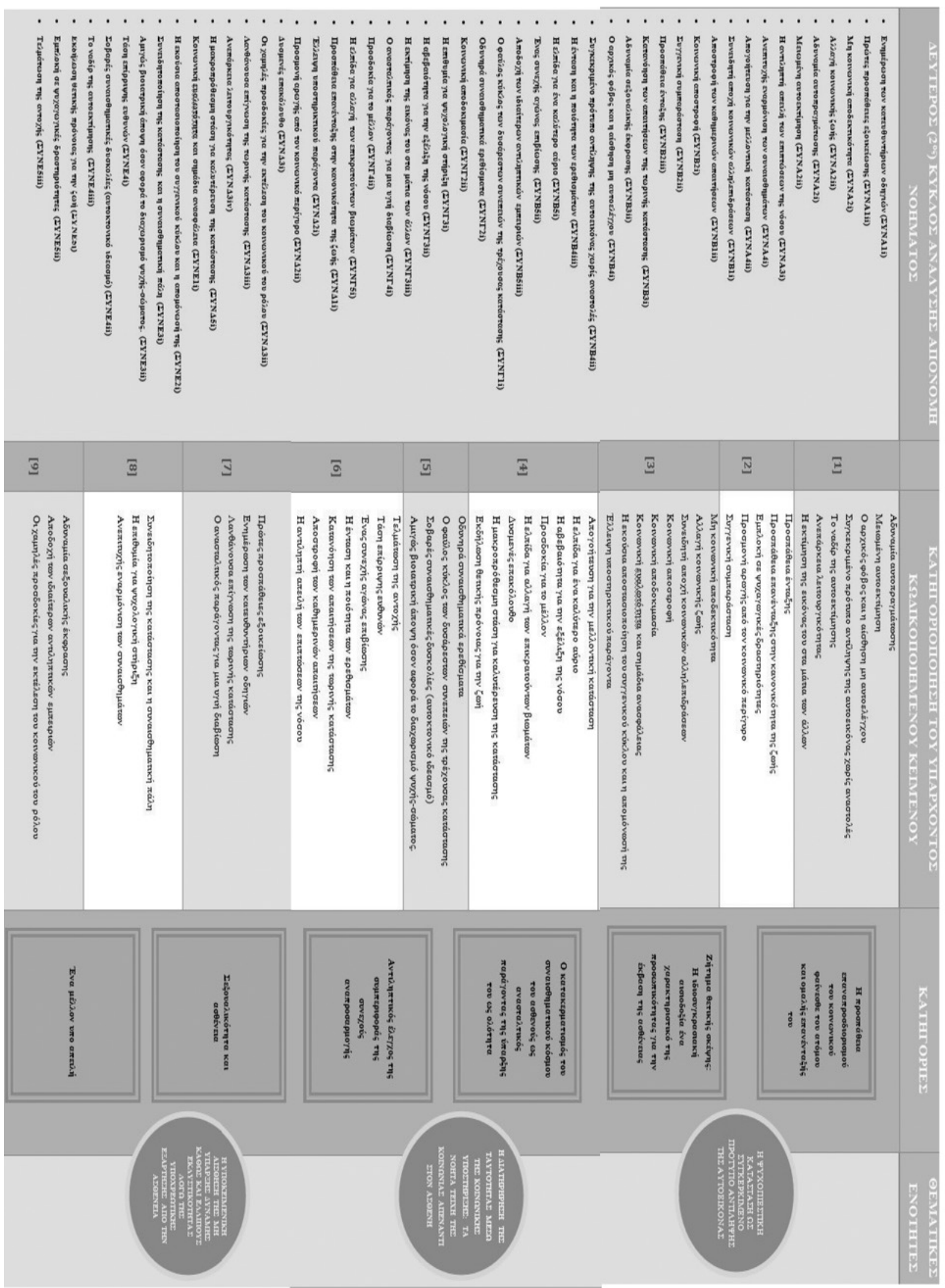

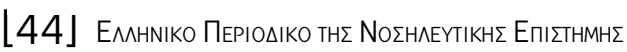




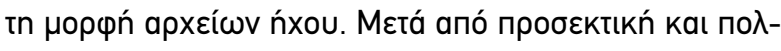

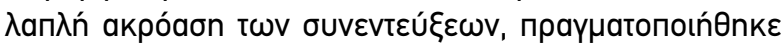

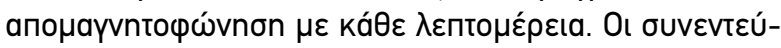

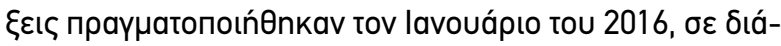

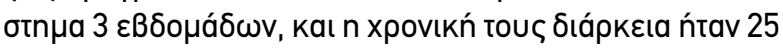
пврі́nоu $\lambda \varepsilon п т$ tá.

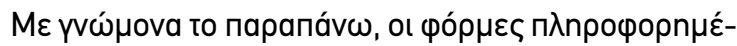

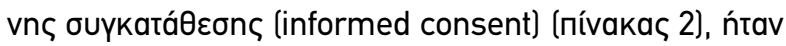

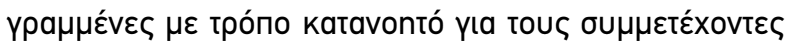

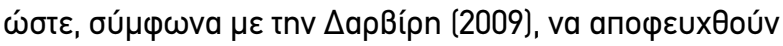

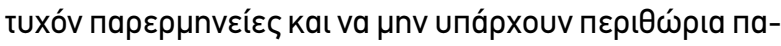

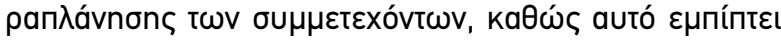

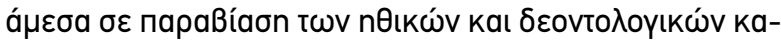

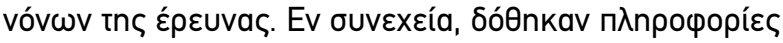

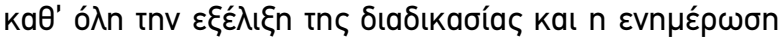

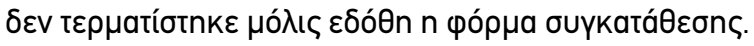

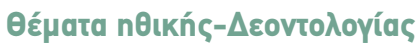

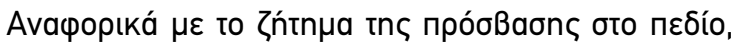

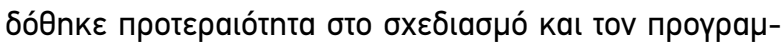

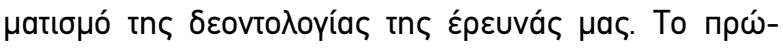

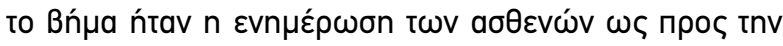

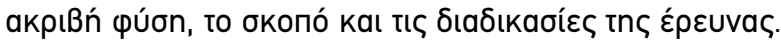

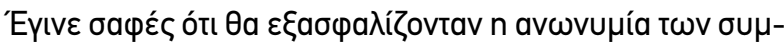

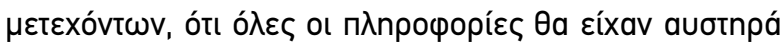

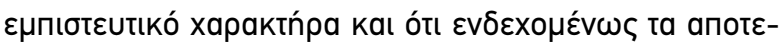
$\lambda \varepsilon ́ \sigma \mu a t a$ va on

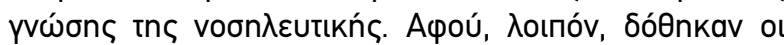

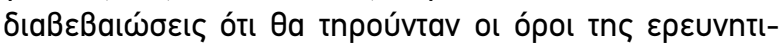

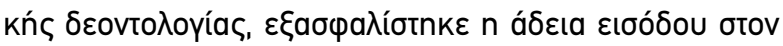

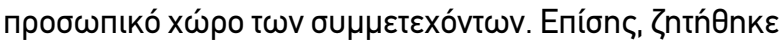

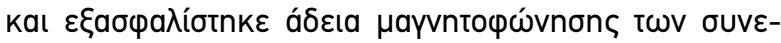

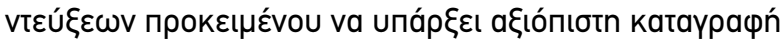

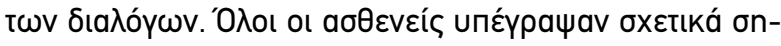

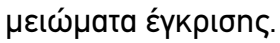

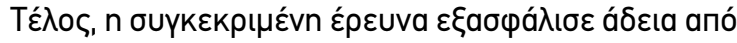

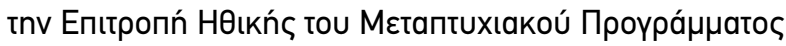

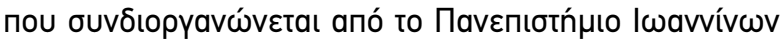

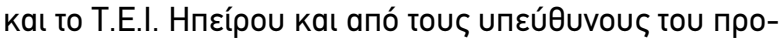

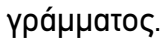

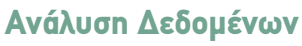

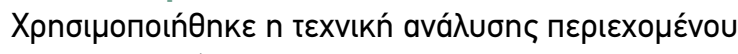
(content analysis), katá Mayring, nou okonós tnç zívaı n

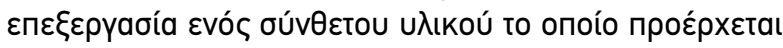

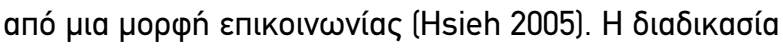

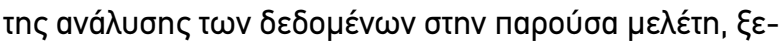

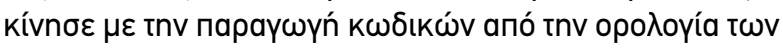

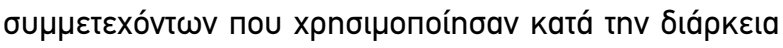

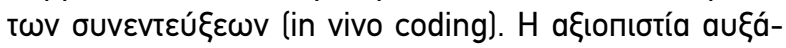

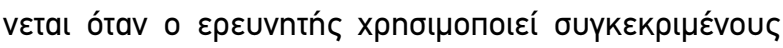

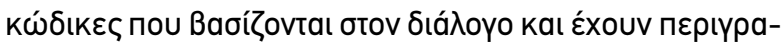

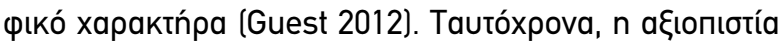

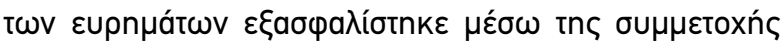

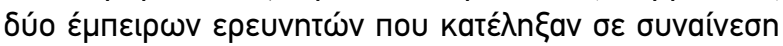

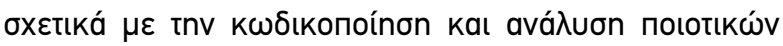

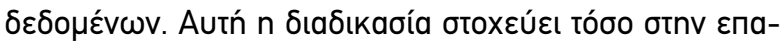

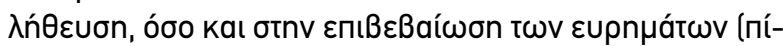
vakas 3$)$.

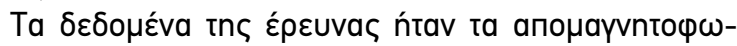

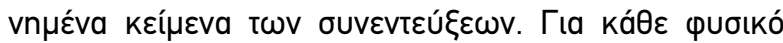

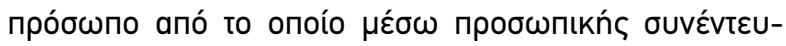

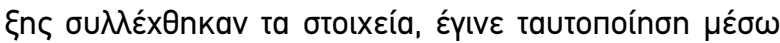

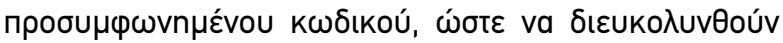

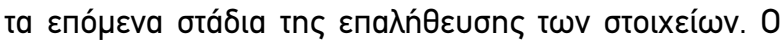

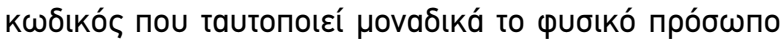

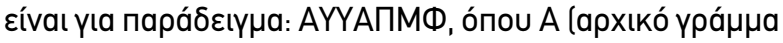

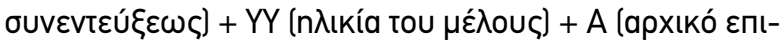

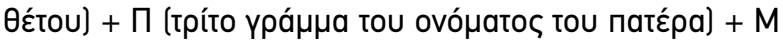

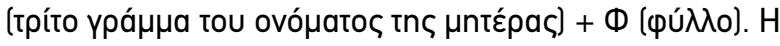

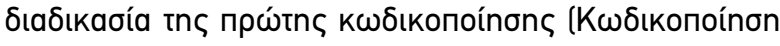

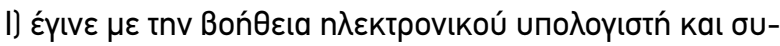

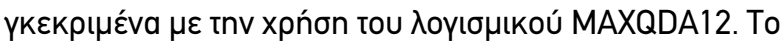

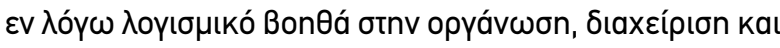

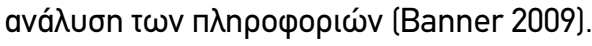

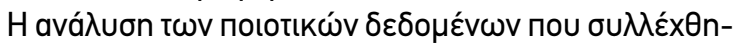

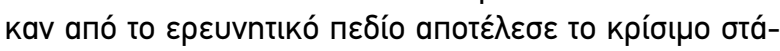

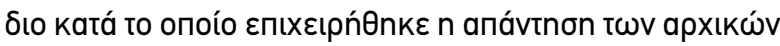

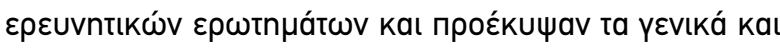

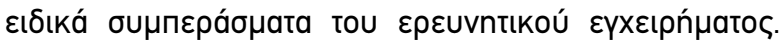

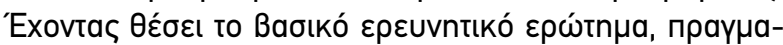

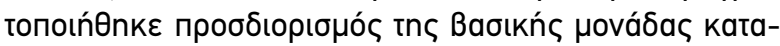

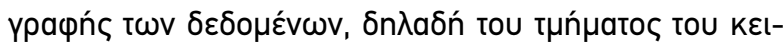

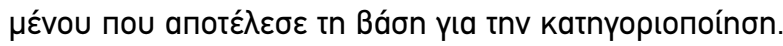

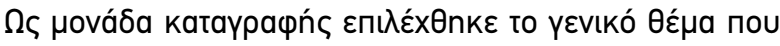

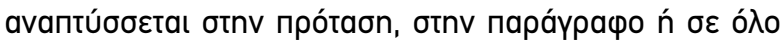

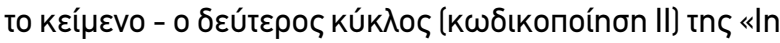
Vivo» texVIkń

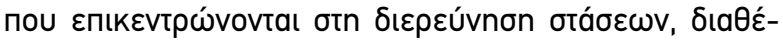

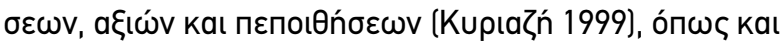

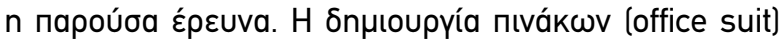

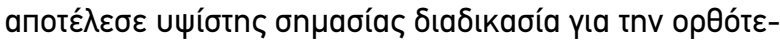

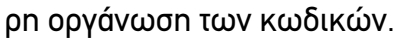

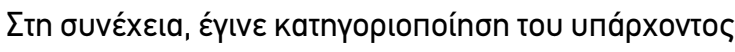

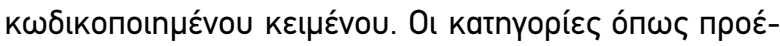

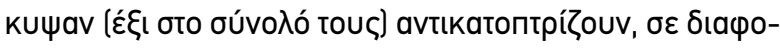

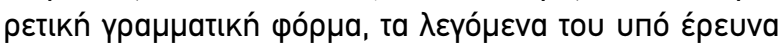

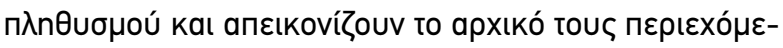

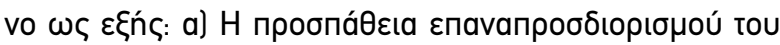

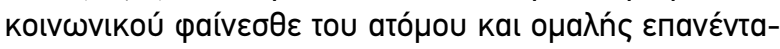

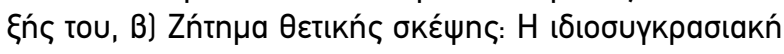

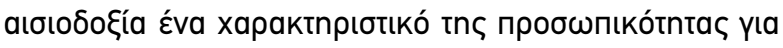

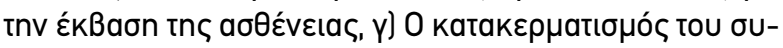

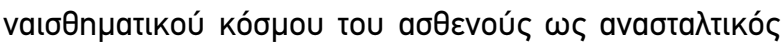

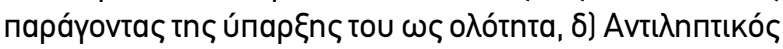

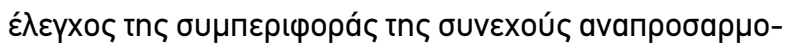




\section{EPEYNHTIKA APOPA}

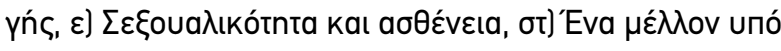

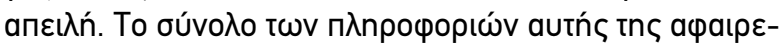

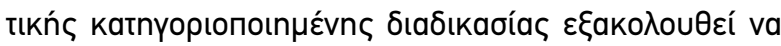

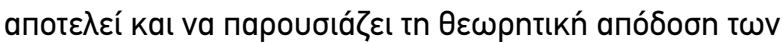

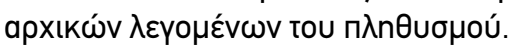

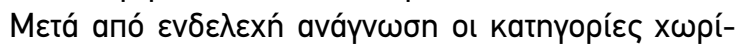

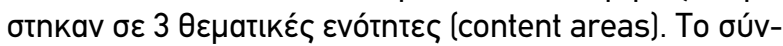

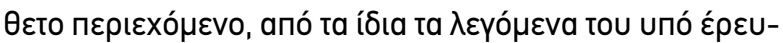

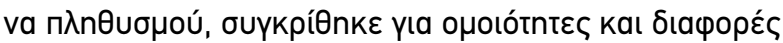

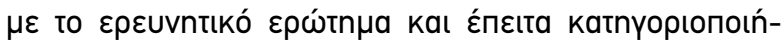

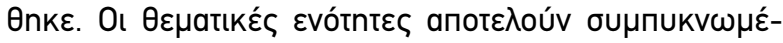

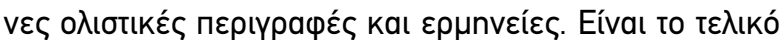

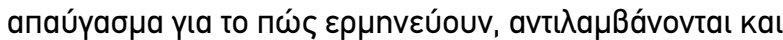

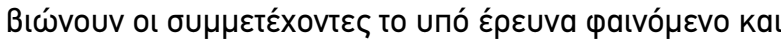

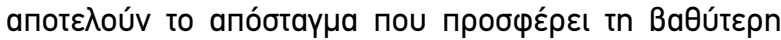

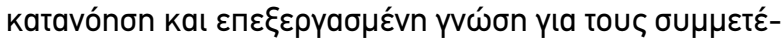
xovtєৎ (Strauss 1998).

\section{АПОТЕАЕГMATA}

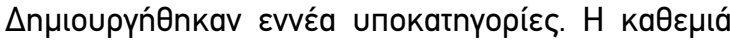

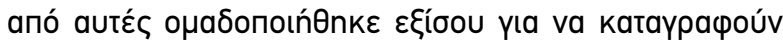

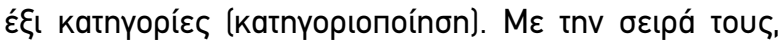

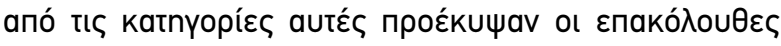

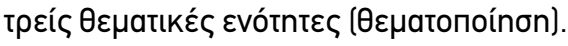

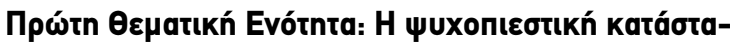

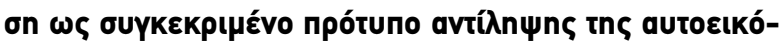
vas

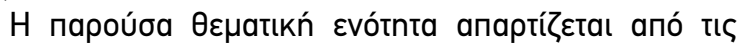

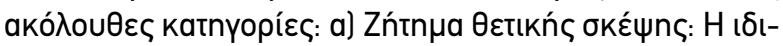

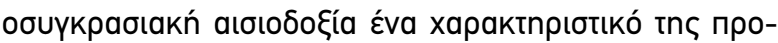

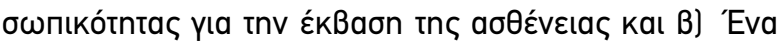

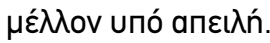

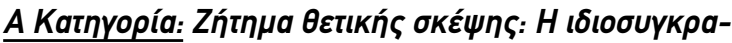

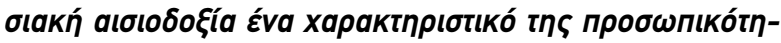

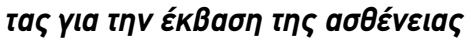

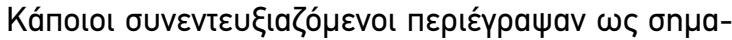

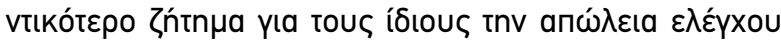

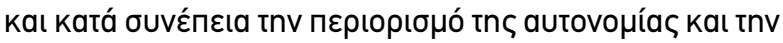

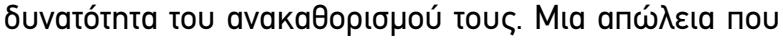

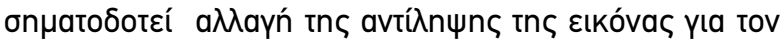

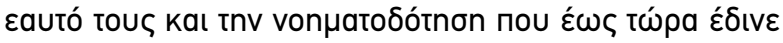

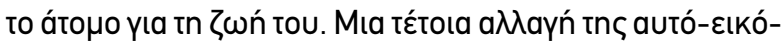

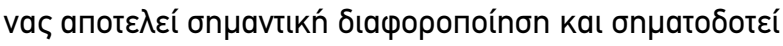

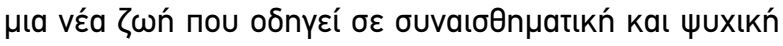

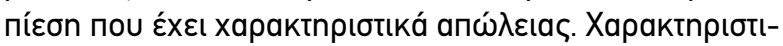

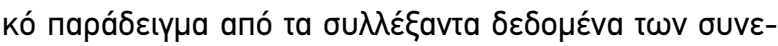

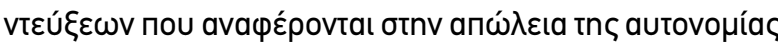

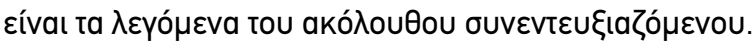

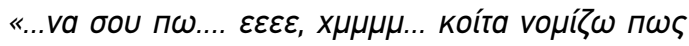

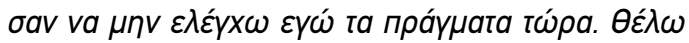

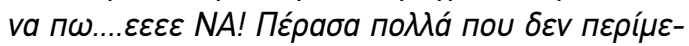

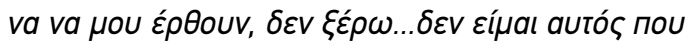

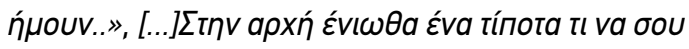

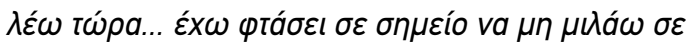

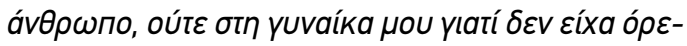

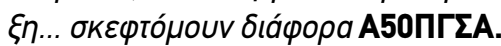

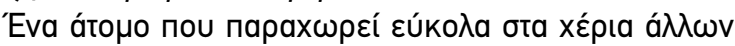

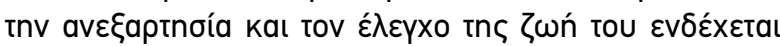

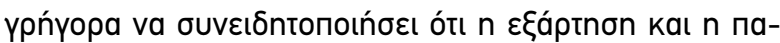

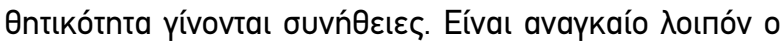

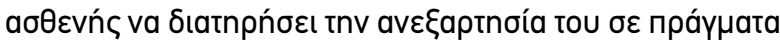

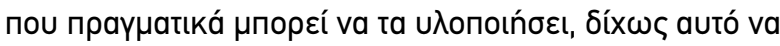

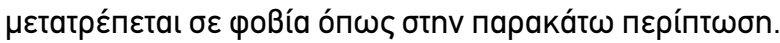

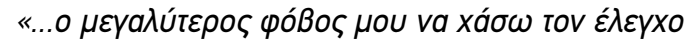

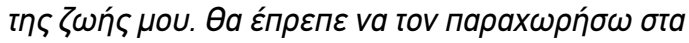

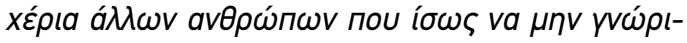

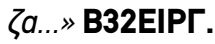

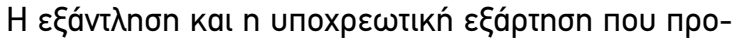

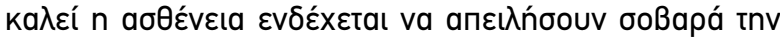

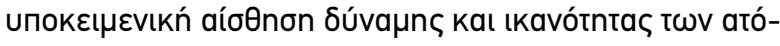

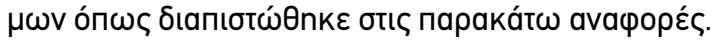

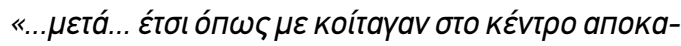

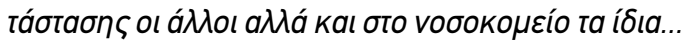

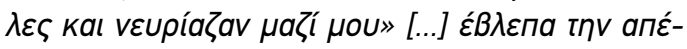

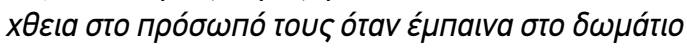
Г58NKГA.

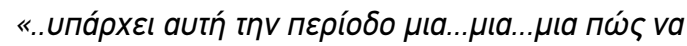

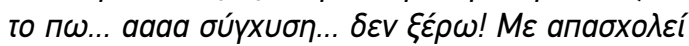

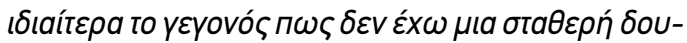

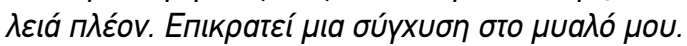

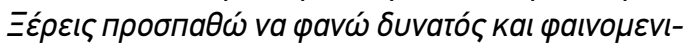

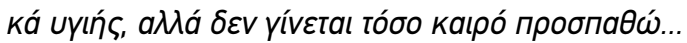

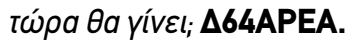

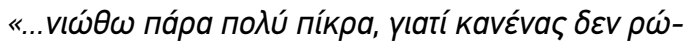

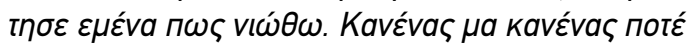

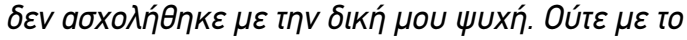

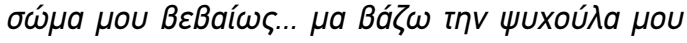
прஸ́та...» E48ZPПГ.

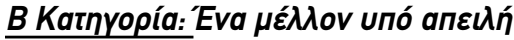

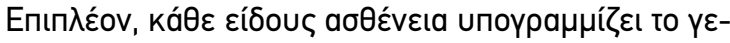

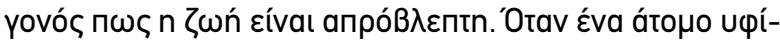

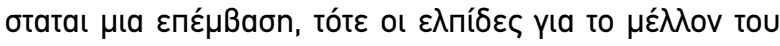

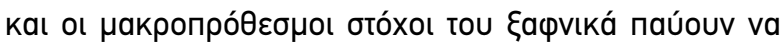

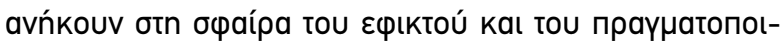

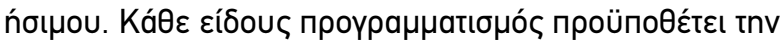

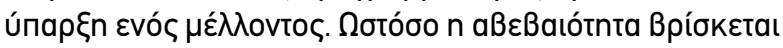

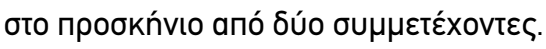

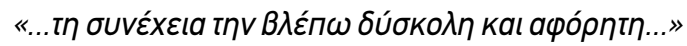
A50חГटA.

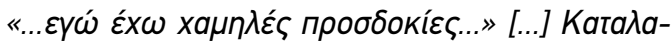

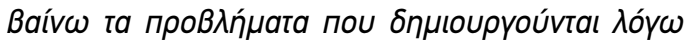

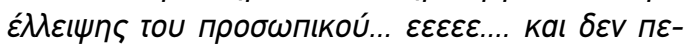

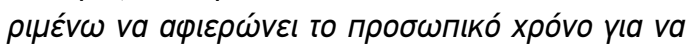




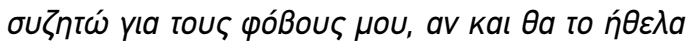
Г58NKГA.

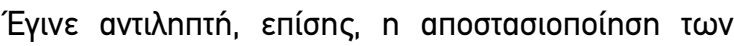

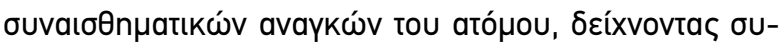

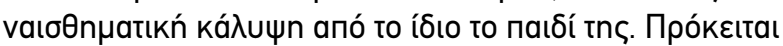

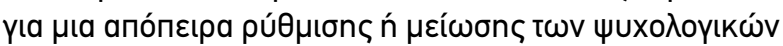

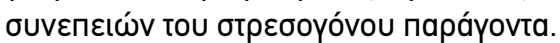

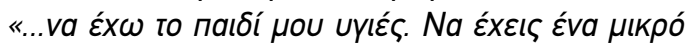

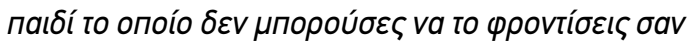

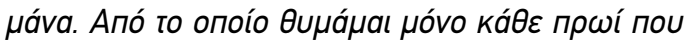

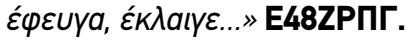

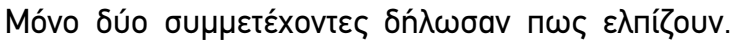

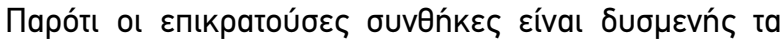

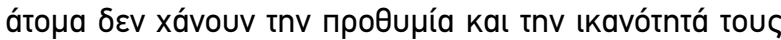

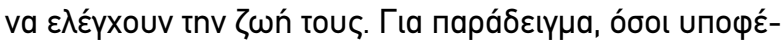

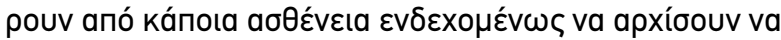

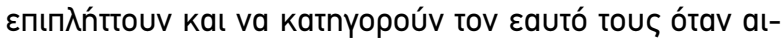

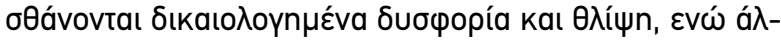

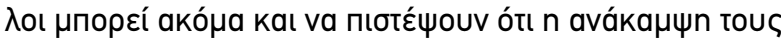

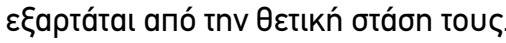

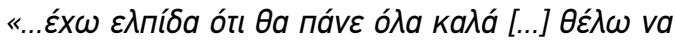

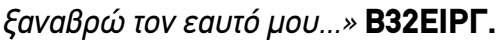

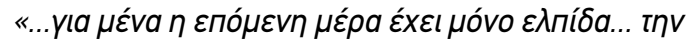

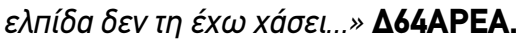

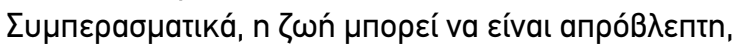

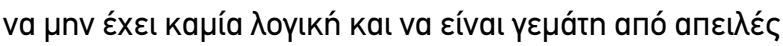

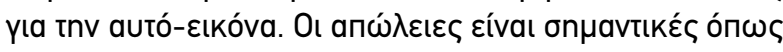

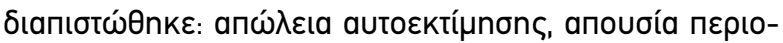

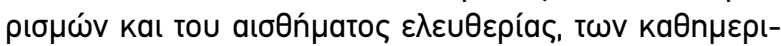

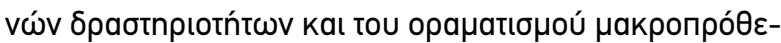

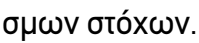

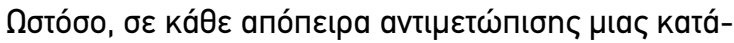

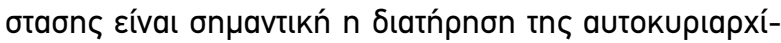

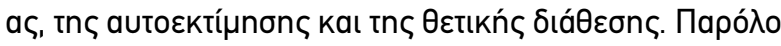

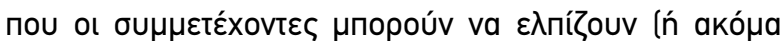

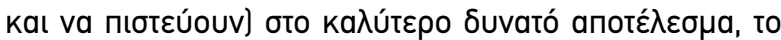

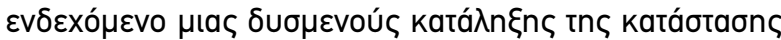

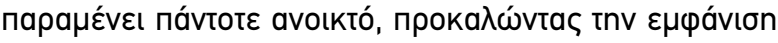
áyxous kat póßou.

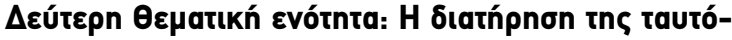

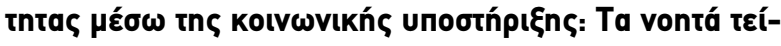

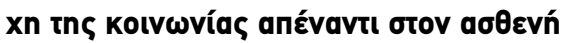

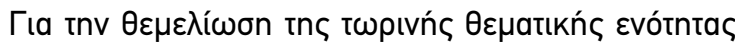

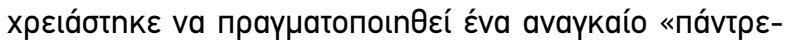

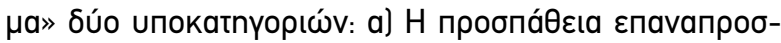

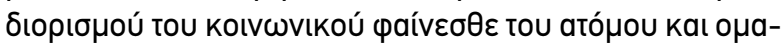

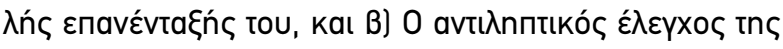

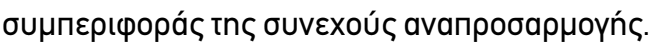

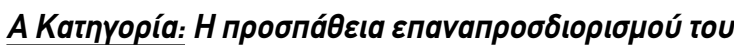

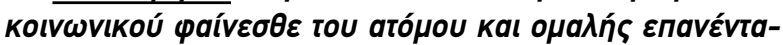
छ̇́s tou

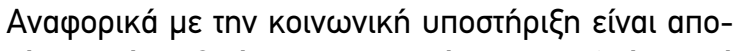

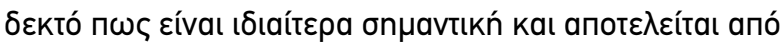

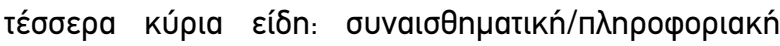

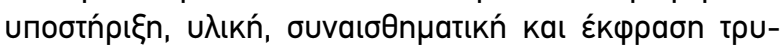

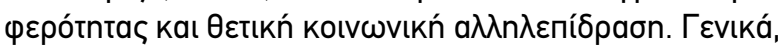

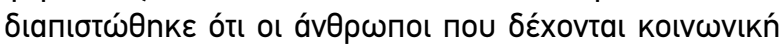

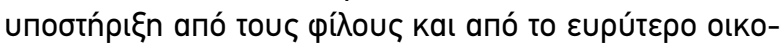

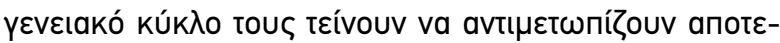

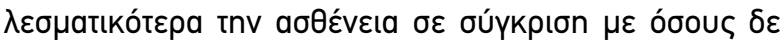

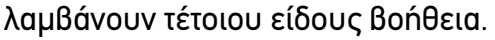

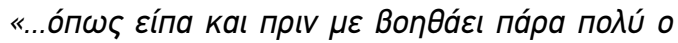

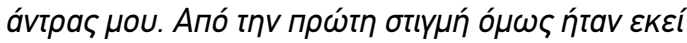

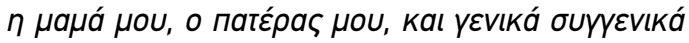
про́б

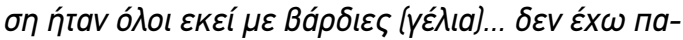

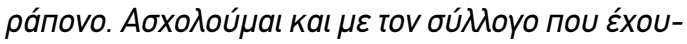

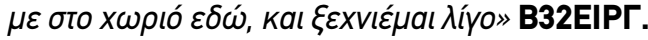

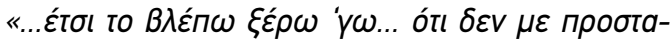

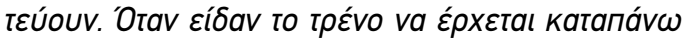

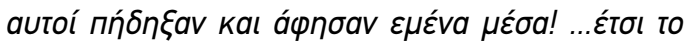

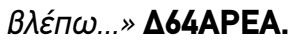

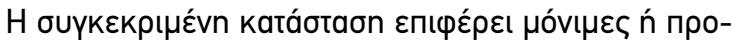

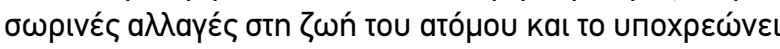

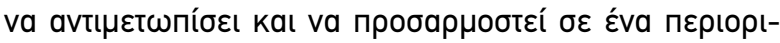

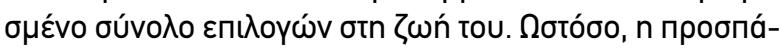

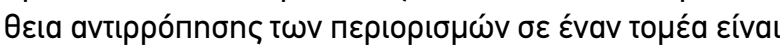

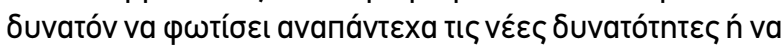

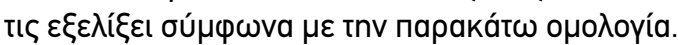

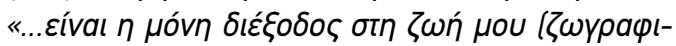

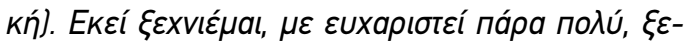

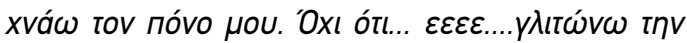

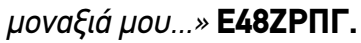

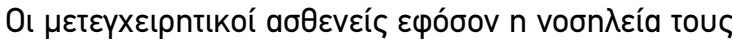

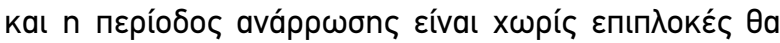

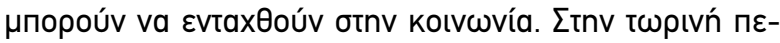

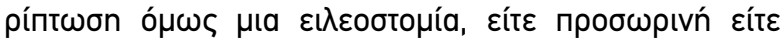

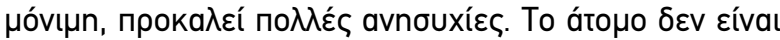

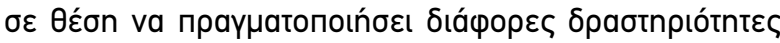

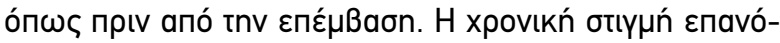

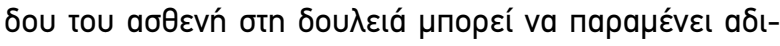

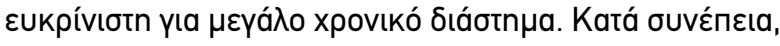

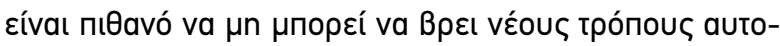

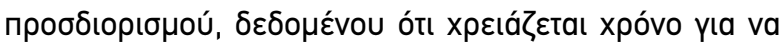

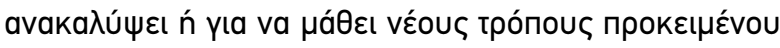

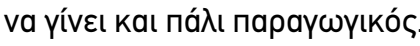

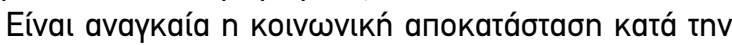

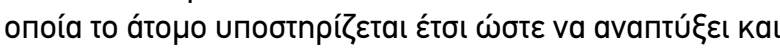

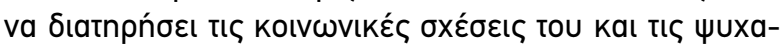

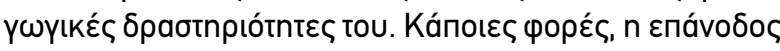

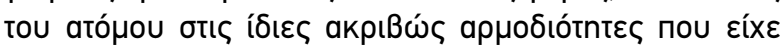

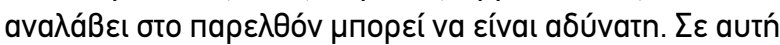

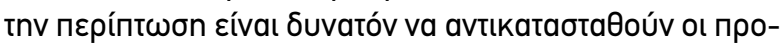

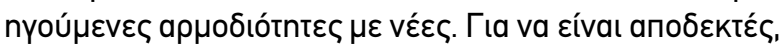




\section{EPEYNHTIKA APӨPA}

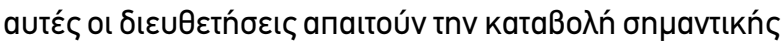

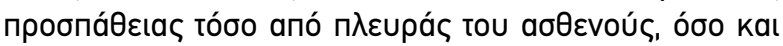

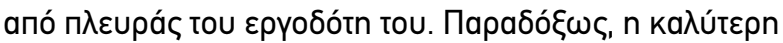

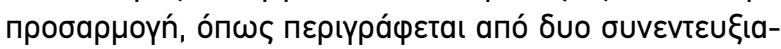

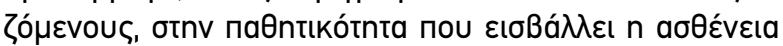

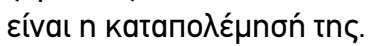

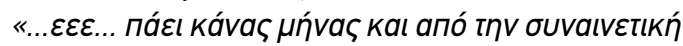

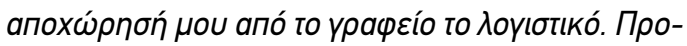

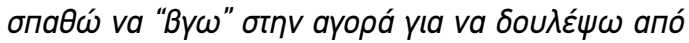

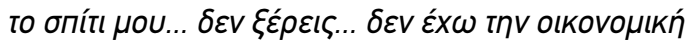

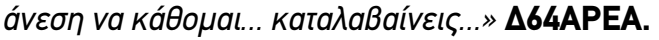

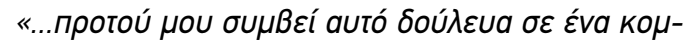

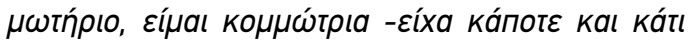

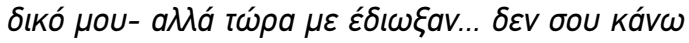

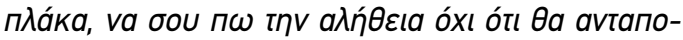

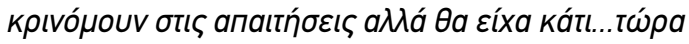

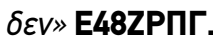

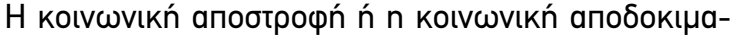

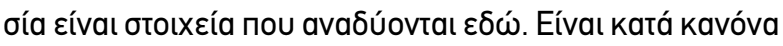

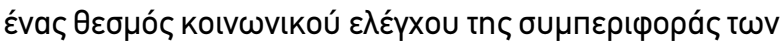

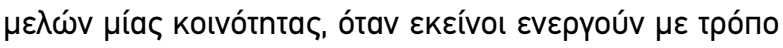

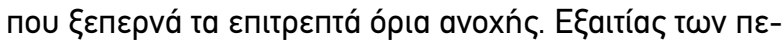

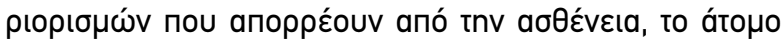

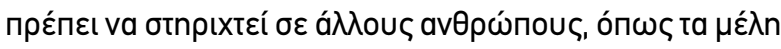

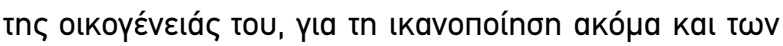

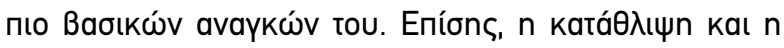

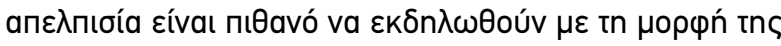

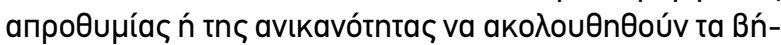

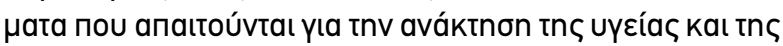

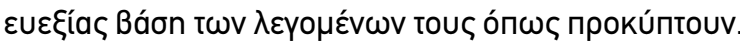

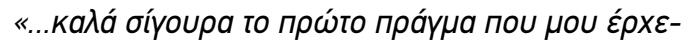

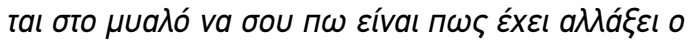

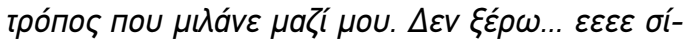

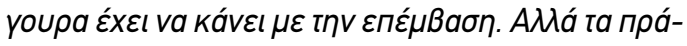

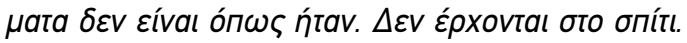

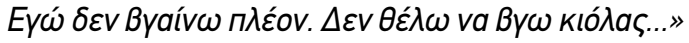
A50ПГЕА.

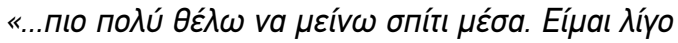

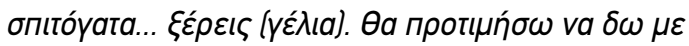

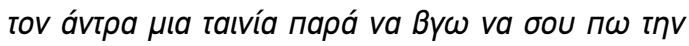

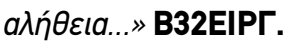

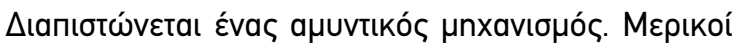

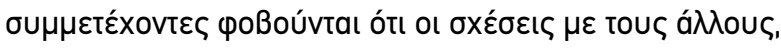

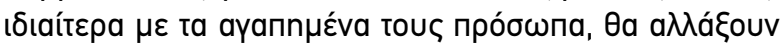

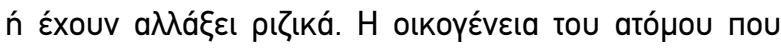

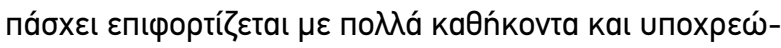

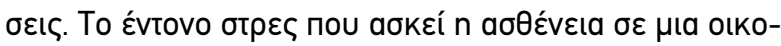

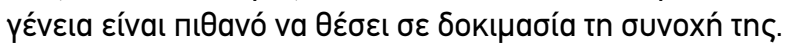

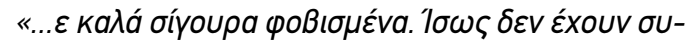

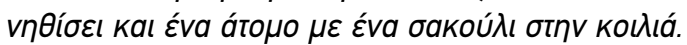

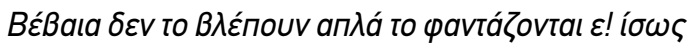

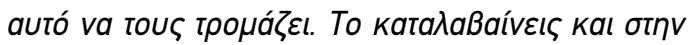

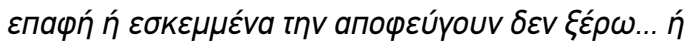

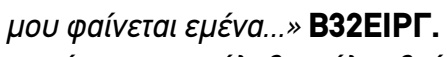

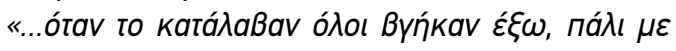

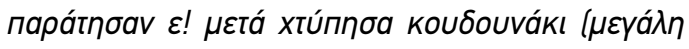

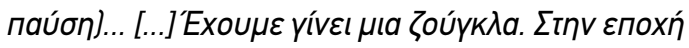

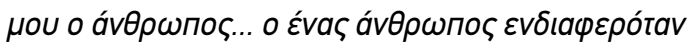

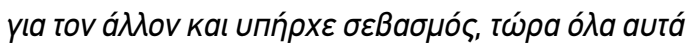

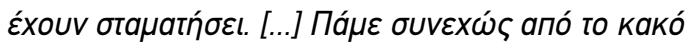

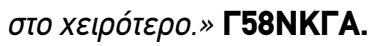

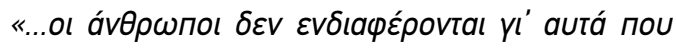

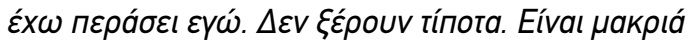

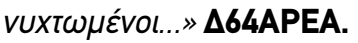

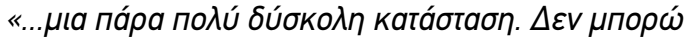

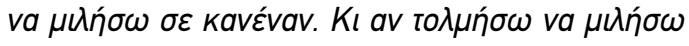

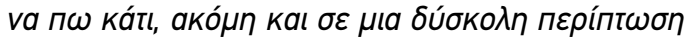

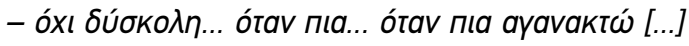

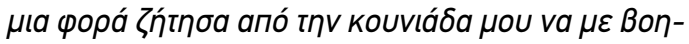

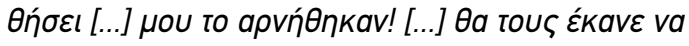

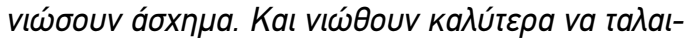

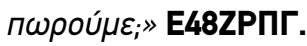

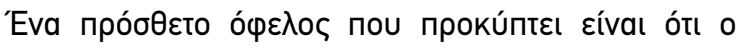

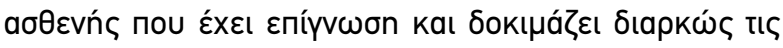

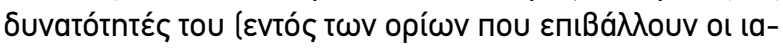

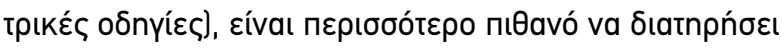

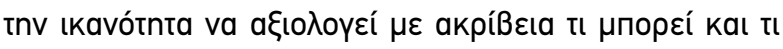

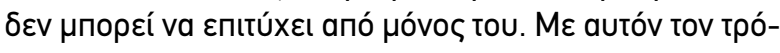

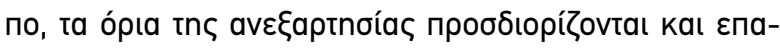

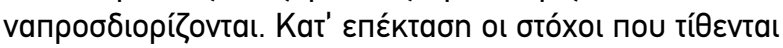

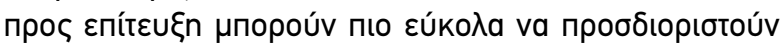

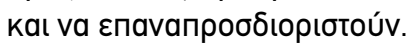

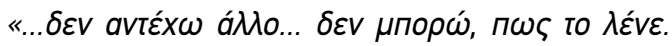

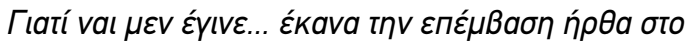

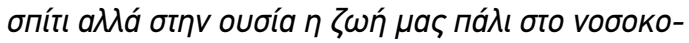

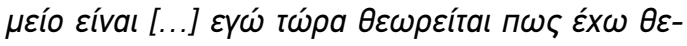

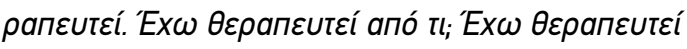

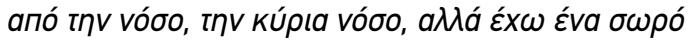

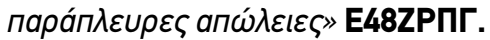

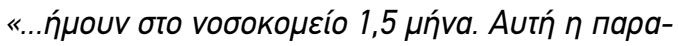

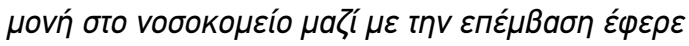

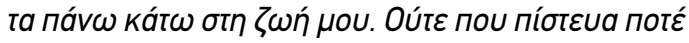

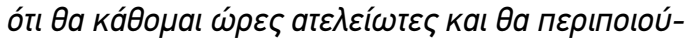

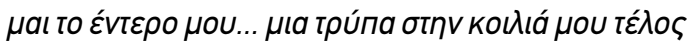

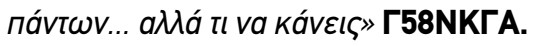

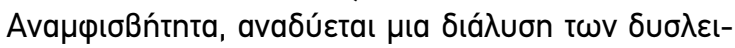

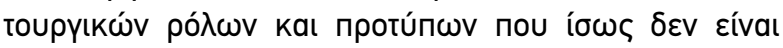

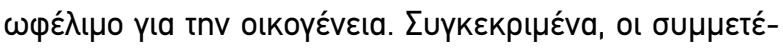

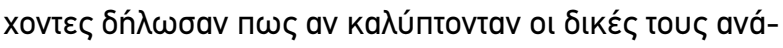

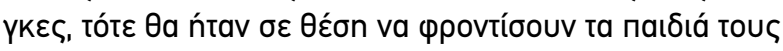

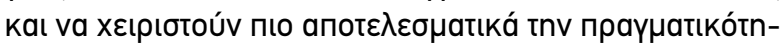

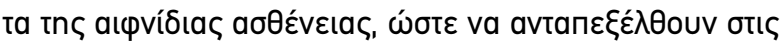

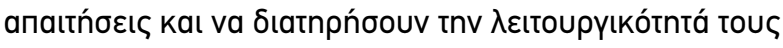

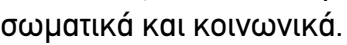

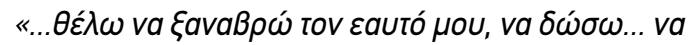

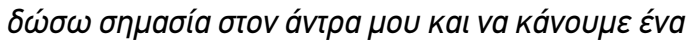




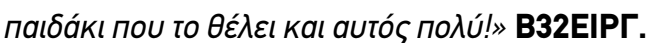

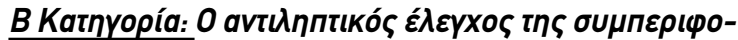

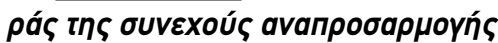

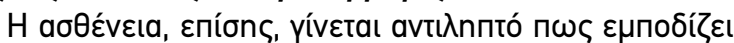

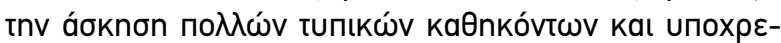

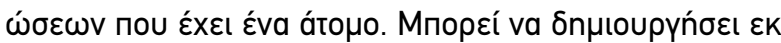

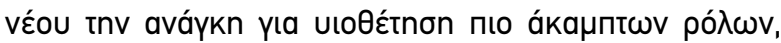

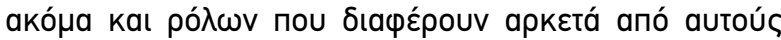

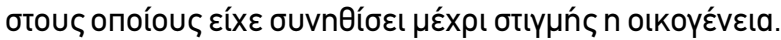

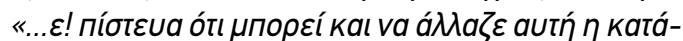

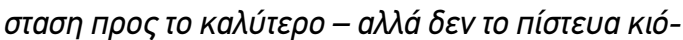

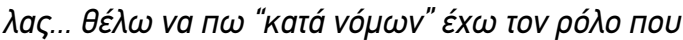

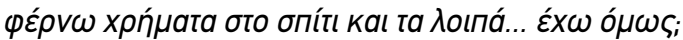

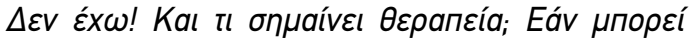

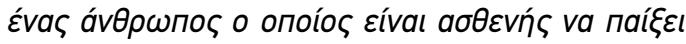

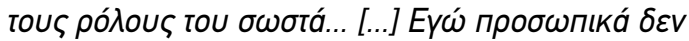

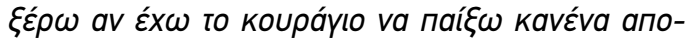

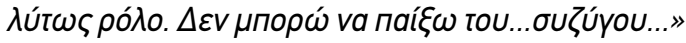
$\triangle 64$ APEA.

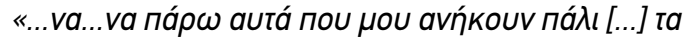

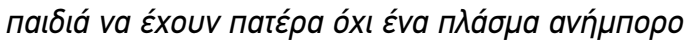

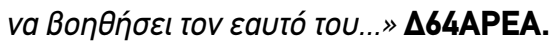

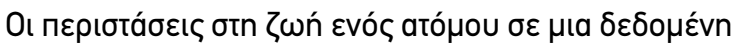

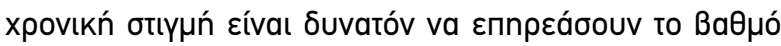

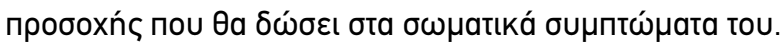

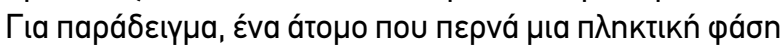

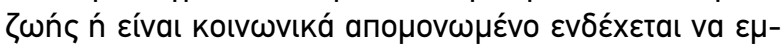

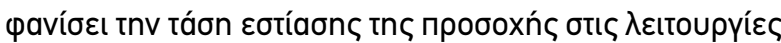

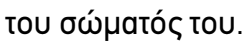

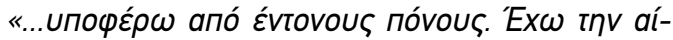

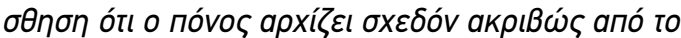

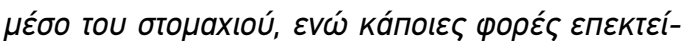

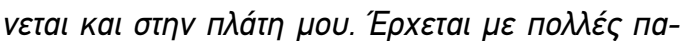

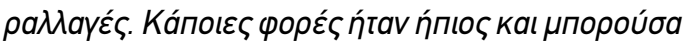

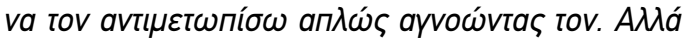

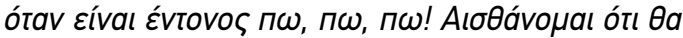

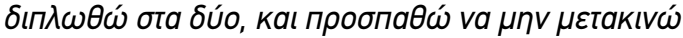

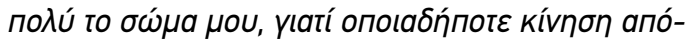

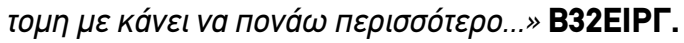
«...

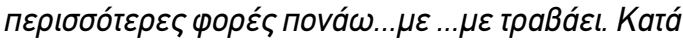

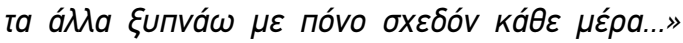
Г58NKГA.

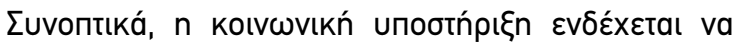

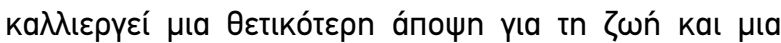

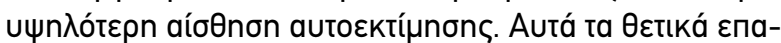

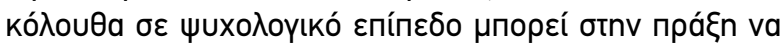

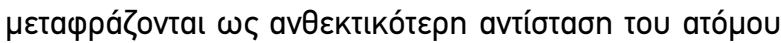

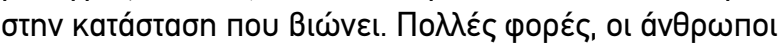

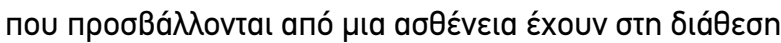

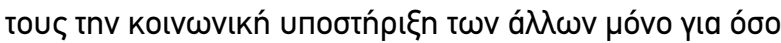

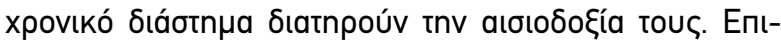

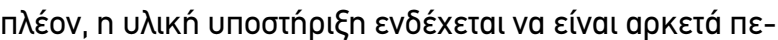

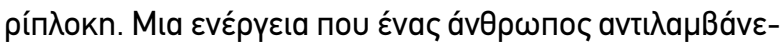

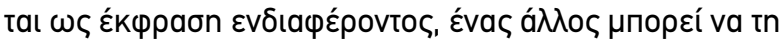

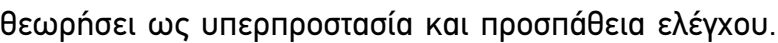

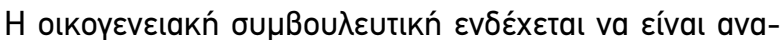

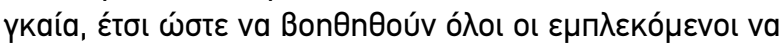

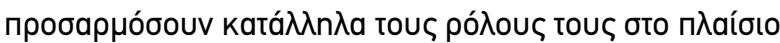

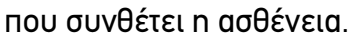

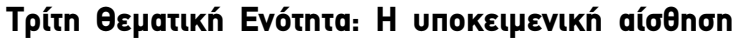

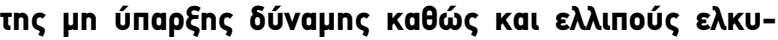

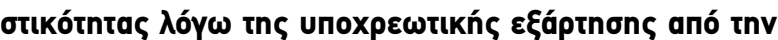

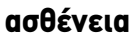

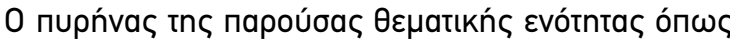

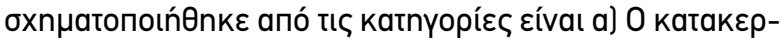

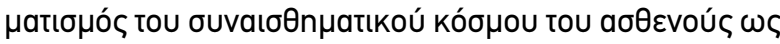

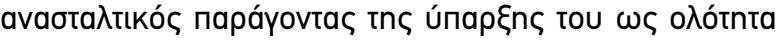

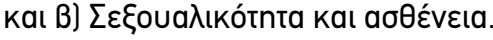

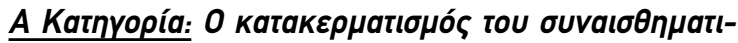

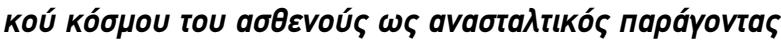

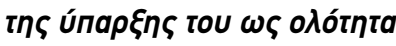

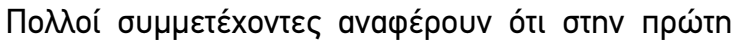

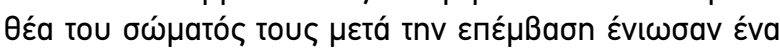

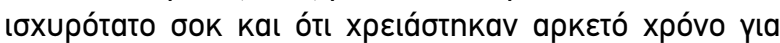

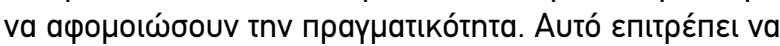

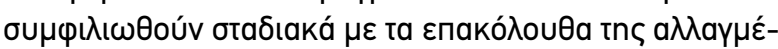

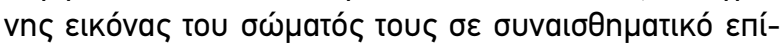

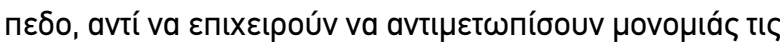

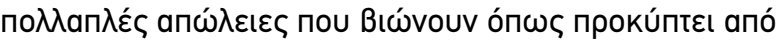

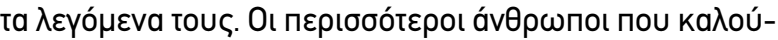

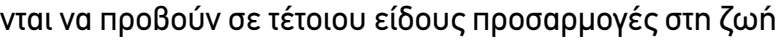

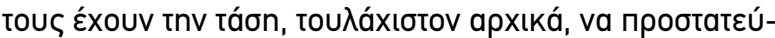

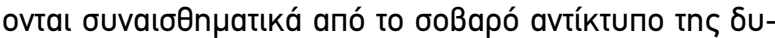

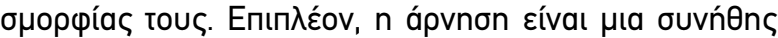

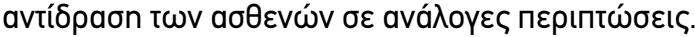

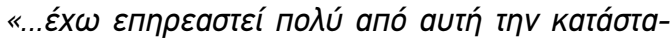

$\sigma \eta \ldots \gg$ A50ПГцA.

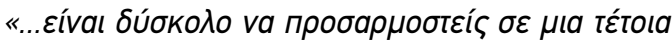

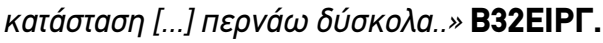

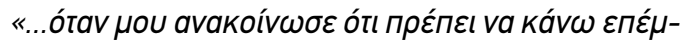

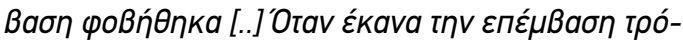
$\mu a \xi a \ldots$. Г58NKГA.

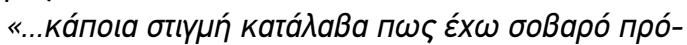

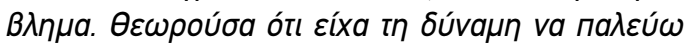

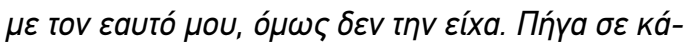

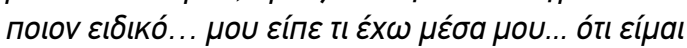

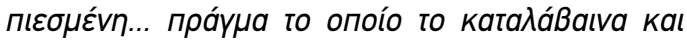

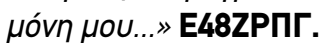

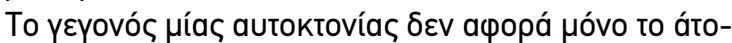

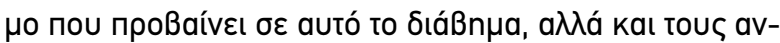

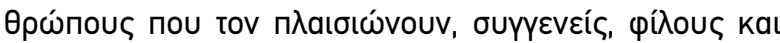

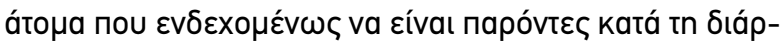




\section{EPEYNHTIKA APӨPA}

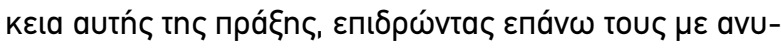

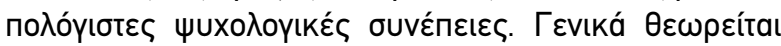

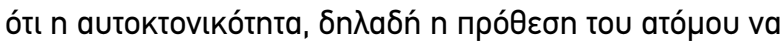

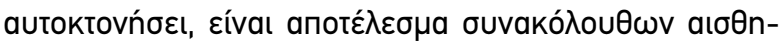

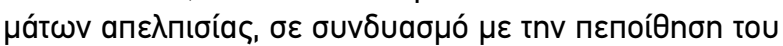

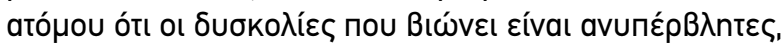

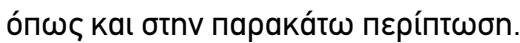

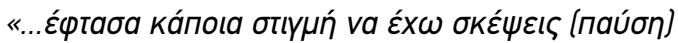

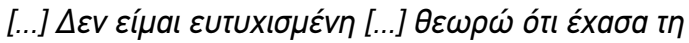

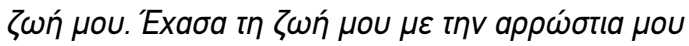

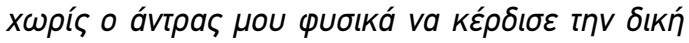
tou...» E48ZPПГ.

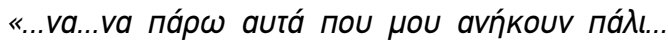

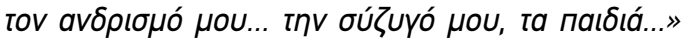
$\triangle 64 A P E A$.

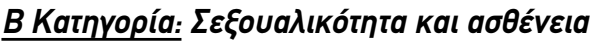

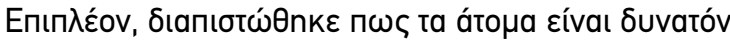

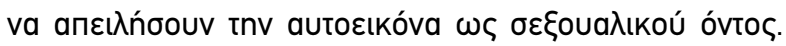

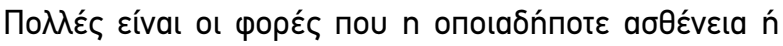

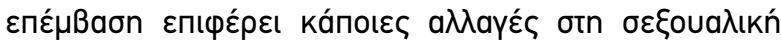

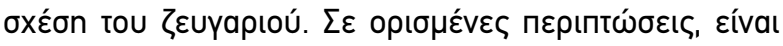

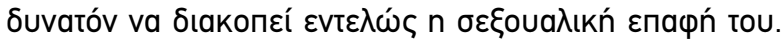

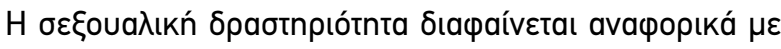

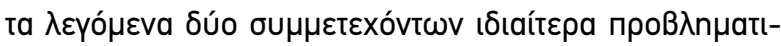

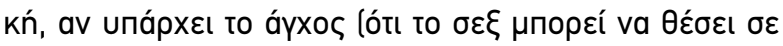

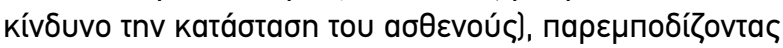

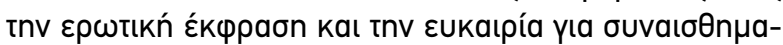
tıkń દYYútnta.

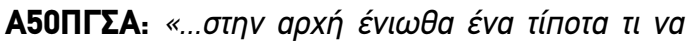

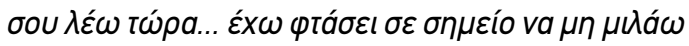

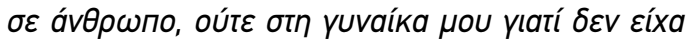

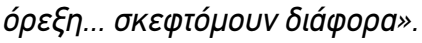

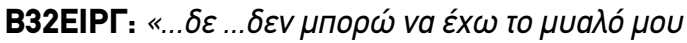

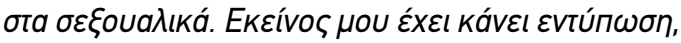

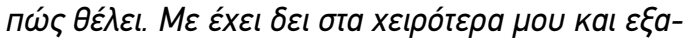

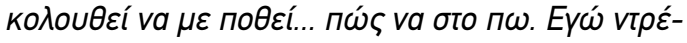

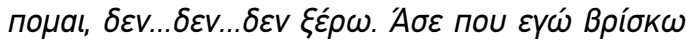

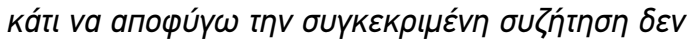

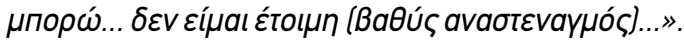

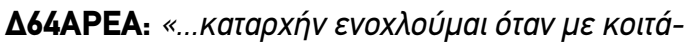

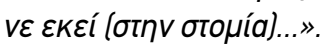

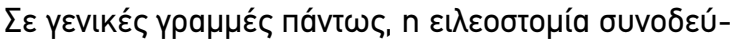

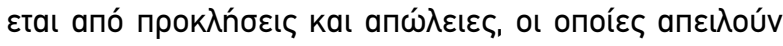

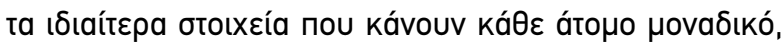

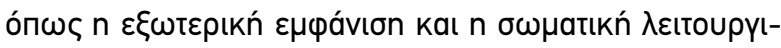

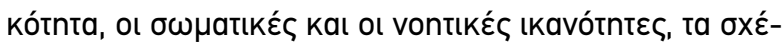

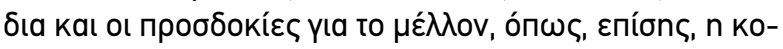

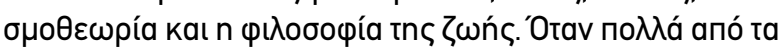

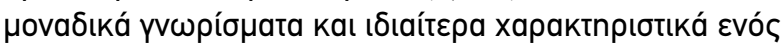

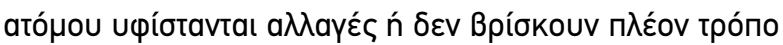

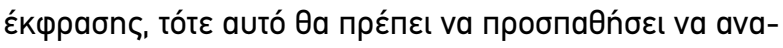

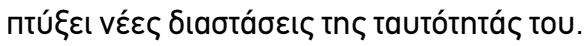

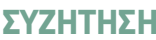

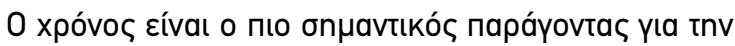

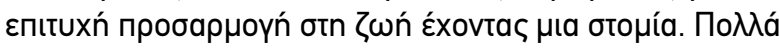

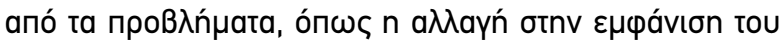

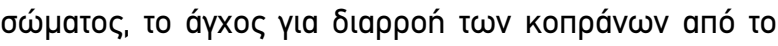

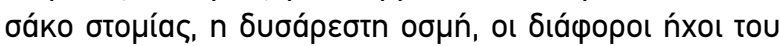

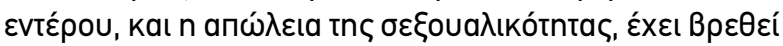

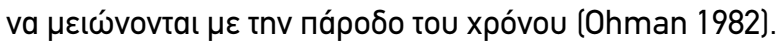

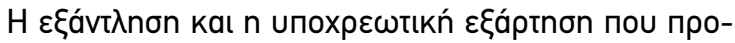

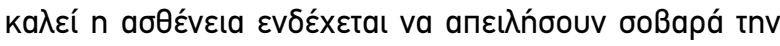

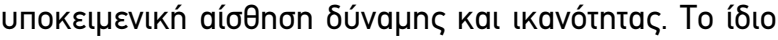

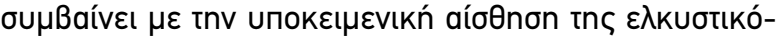

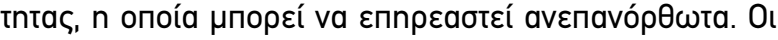

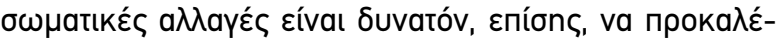

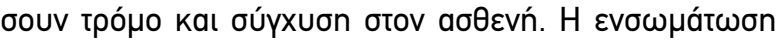

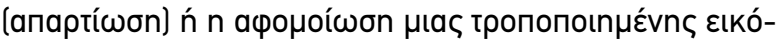

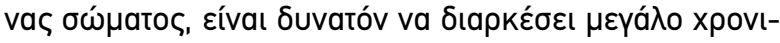

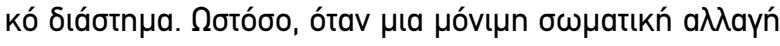

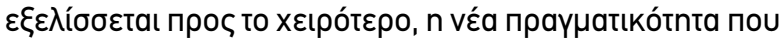

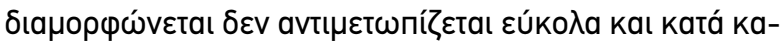
vóva, óxı a

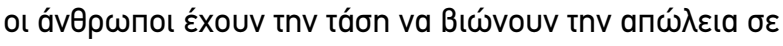

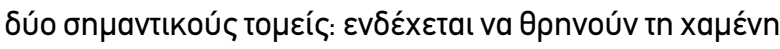

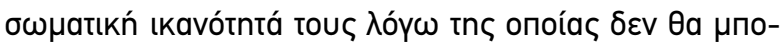

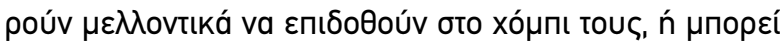

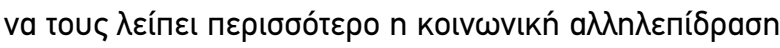

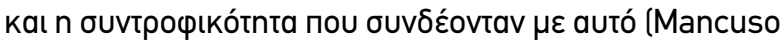
2010).

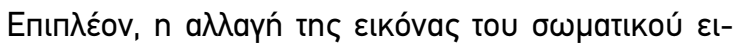

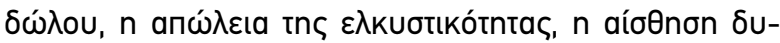

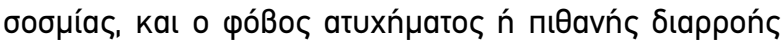

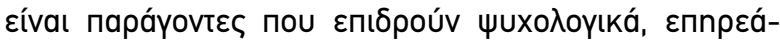

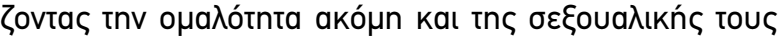

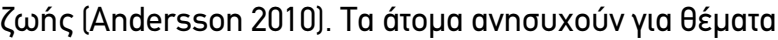

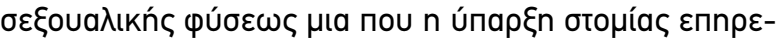

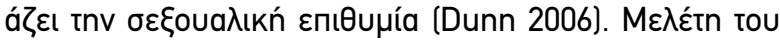

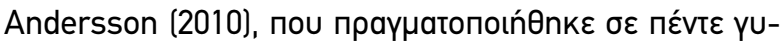

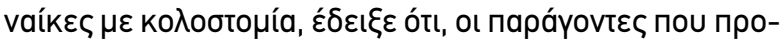

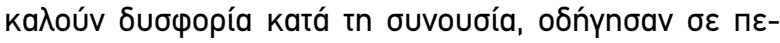

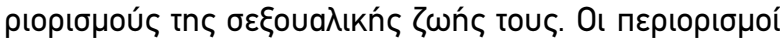

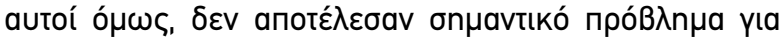

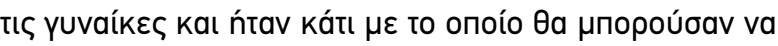

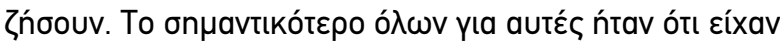

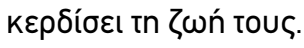

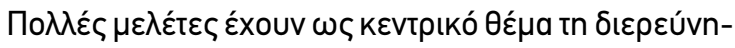

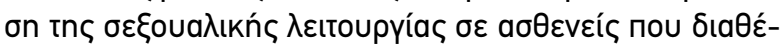

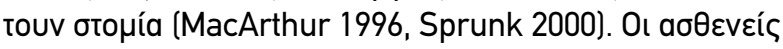

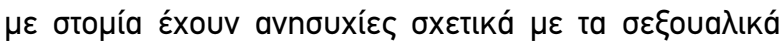

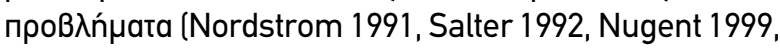

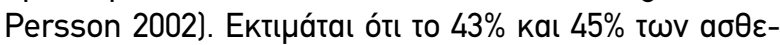

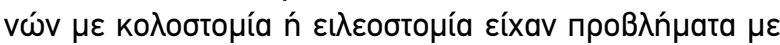

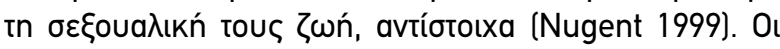

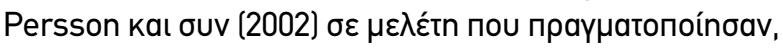




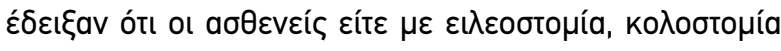

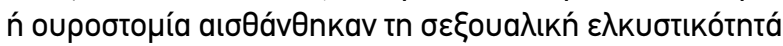

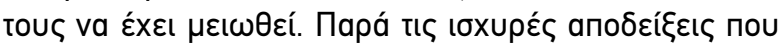

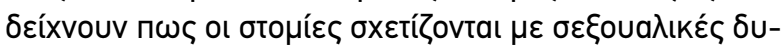

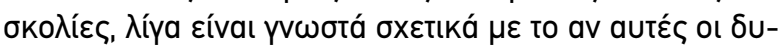

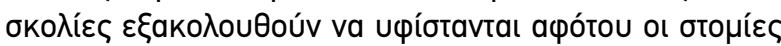
apaip\&Өoúv (Krouse 2009, Kasparek 2012).

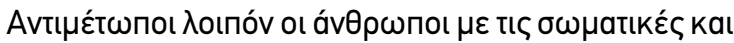

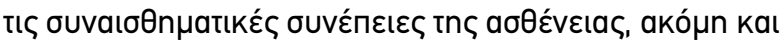

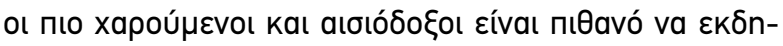

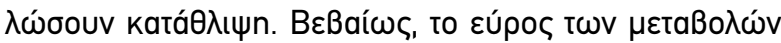

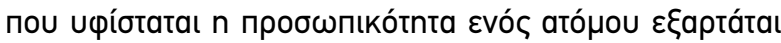

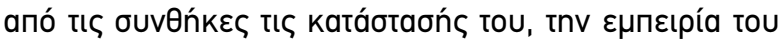

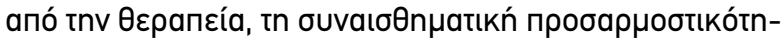

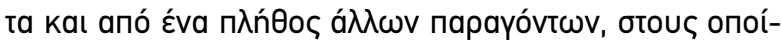

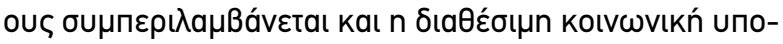
otńpı६n (Marques 2011).

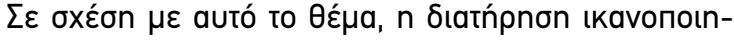

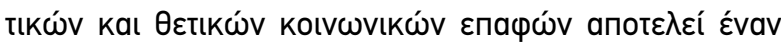

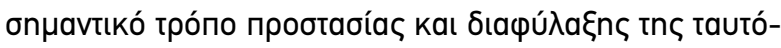

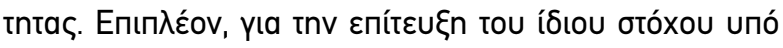

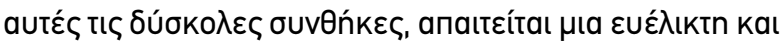

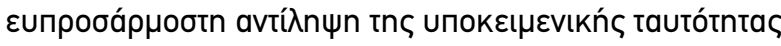

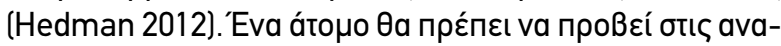

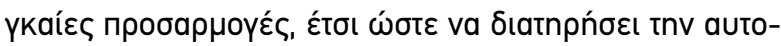

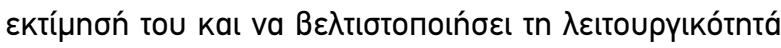

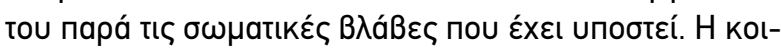

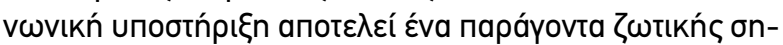

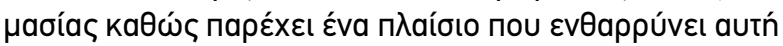

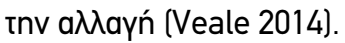

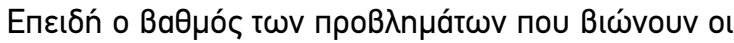

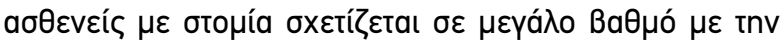

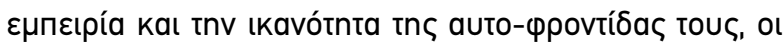

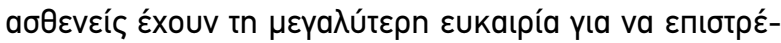

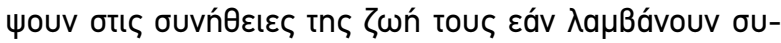

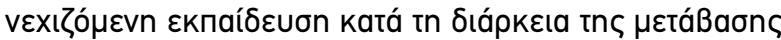

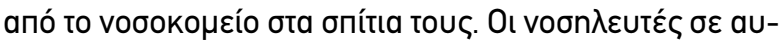

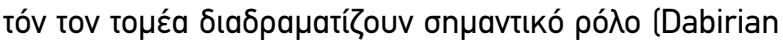
2011).

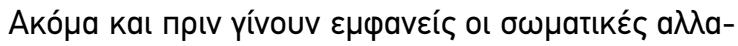

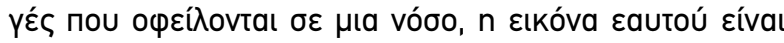

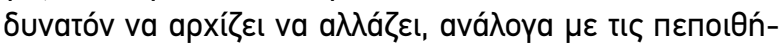

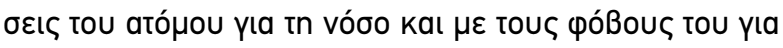

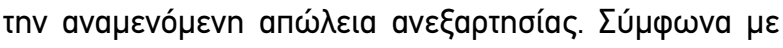

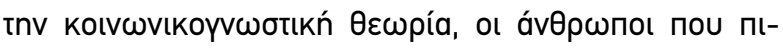

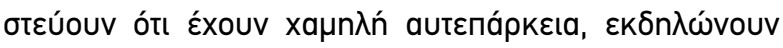

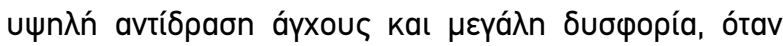

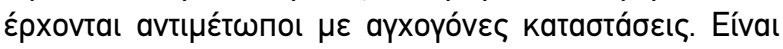

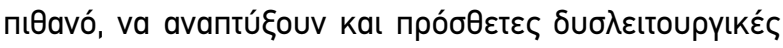

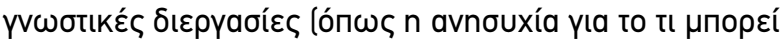

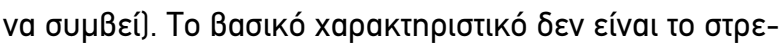

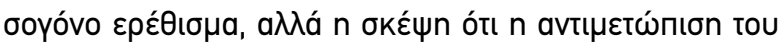

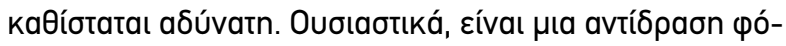

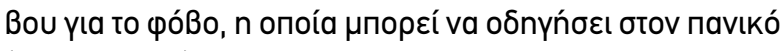

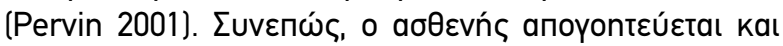

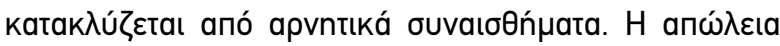

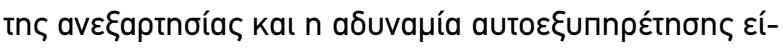

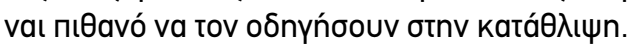

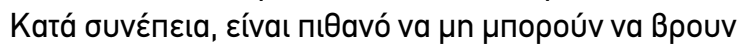

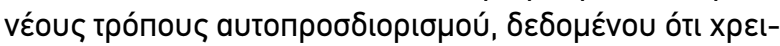

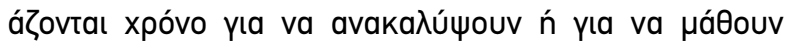

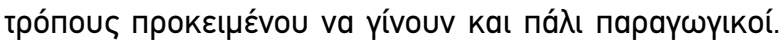

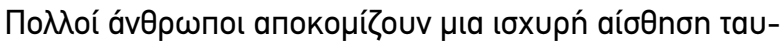

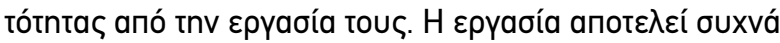

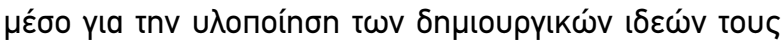

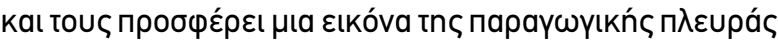

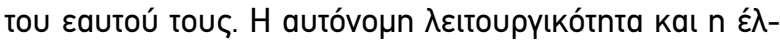

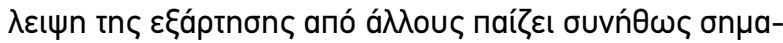

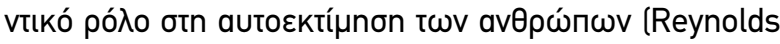

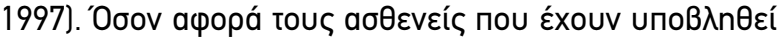

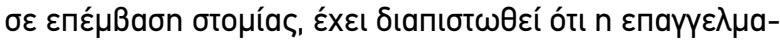

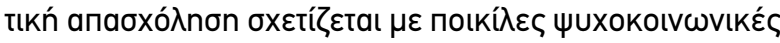

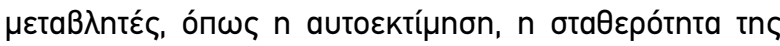

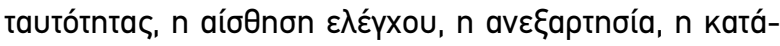

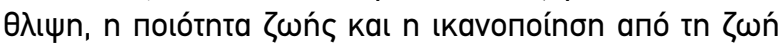
(Duitsman \& Cychosz 1994). Oı ávӨpwnoı nou avńkouv

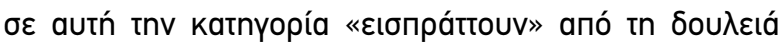

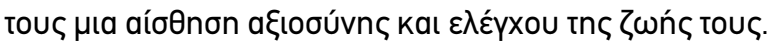

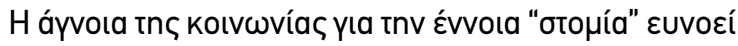

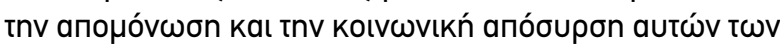

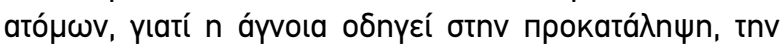

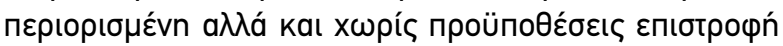

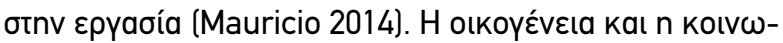

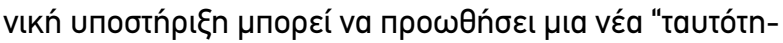

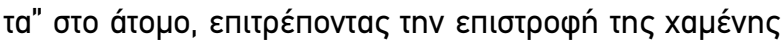

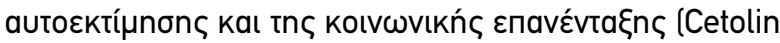

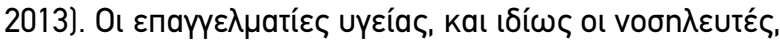

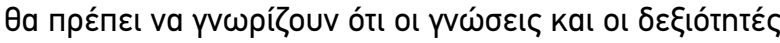

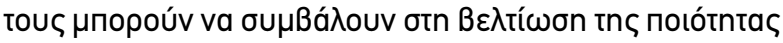

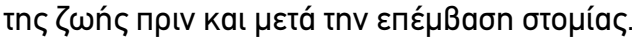

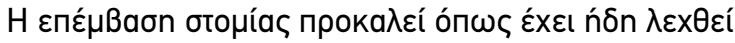

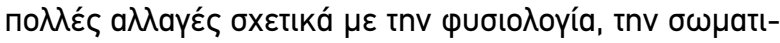

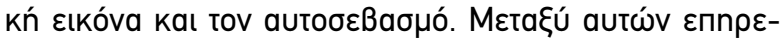

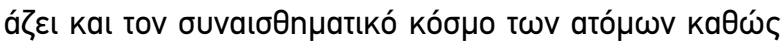

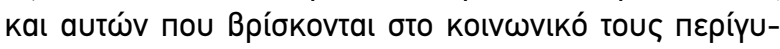

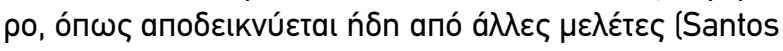
2006, Cascais 2007, Santos 2008). H ouZńtnon nepí au-

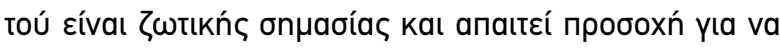

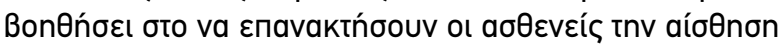

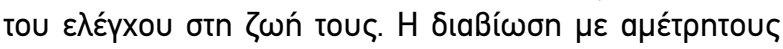

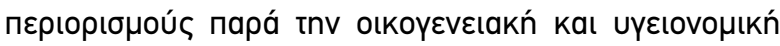

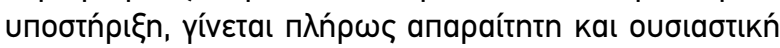

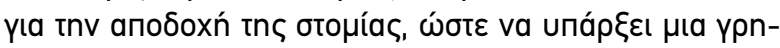

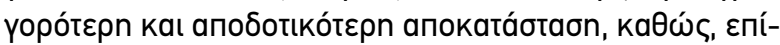

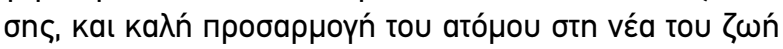
(Backes 2012). 


\section{EPEYNHTIKA APOPA}

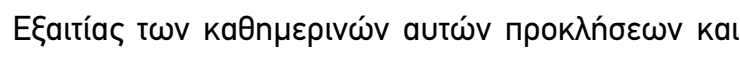

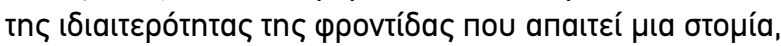

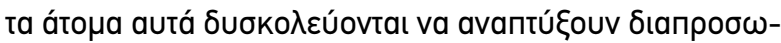

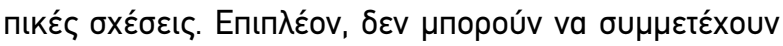

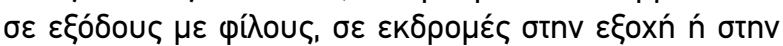

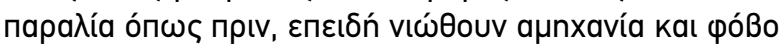

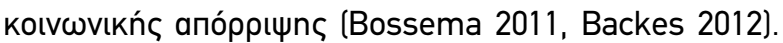

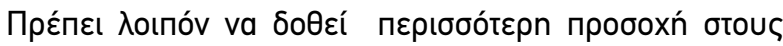

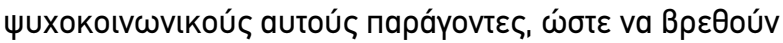

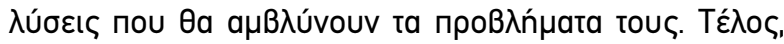

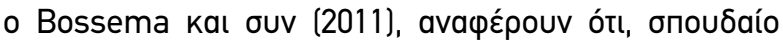

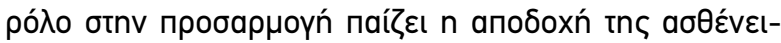

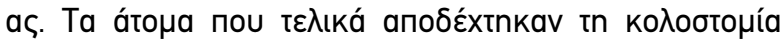

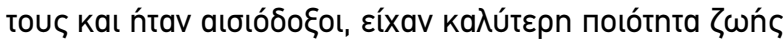

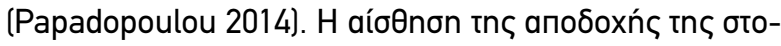

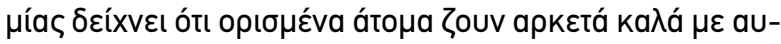

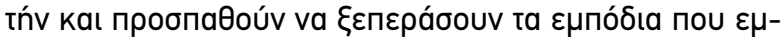

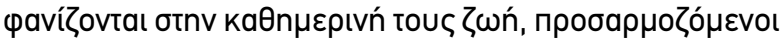

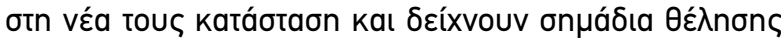

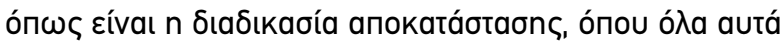

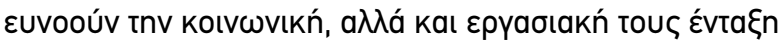
(Recalla 2013).

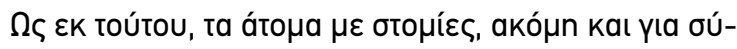

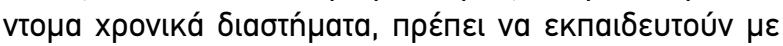

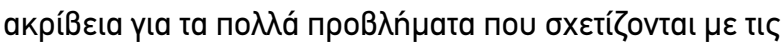

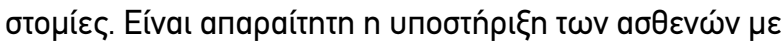

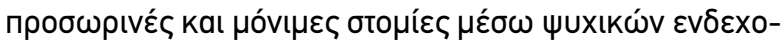

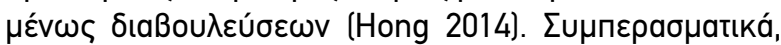

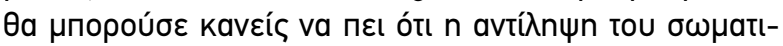

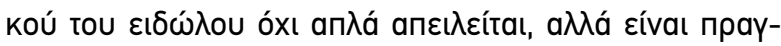

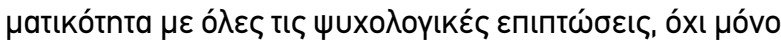

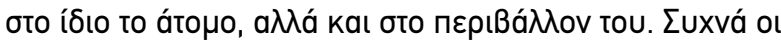

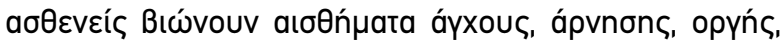

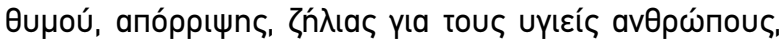

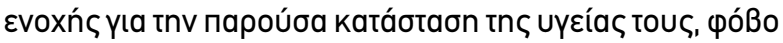

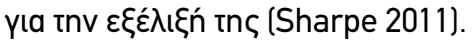

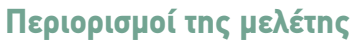

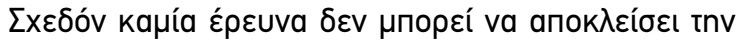

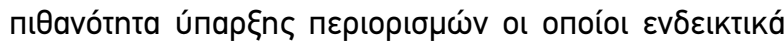

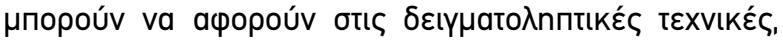

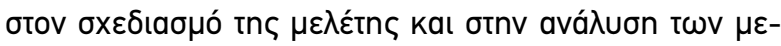

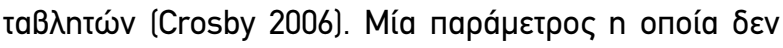

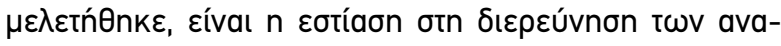

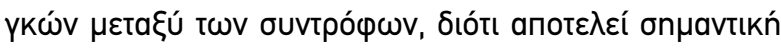

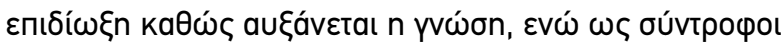

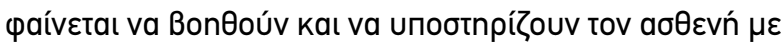

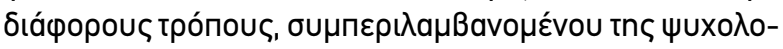
үıkńs otńpı६nç.

\section{¿YMПEPAГMATA}

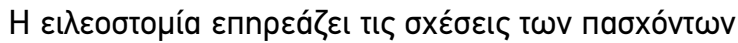

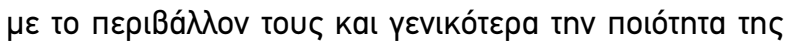

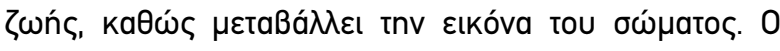

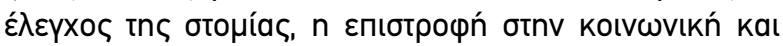

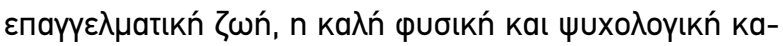

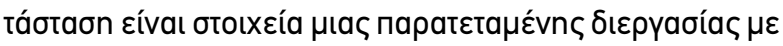

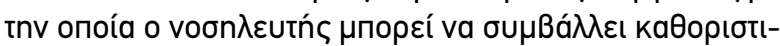

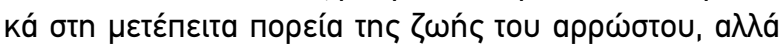

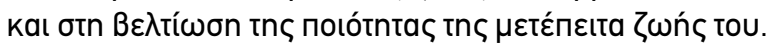

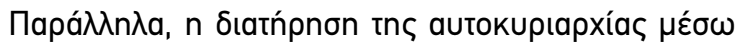

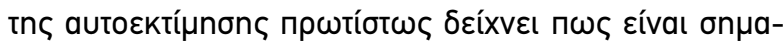

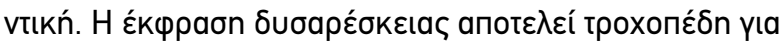

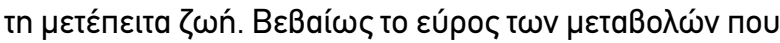

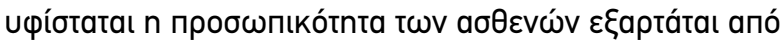

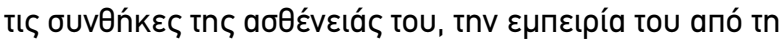

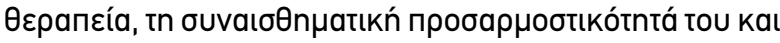

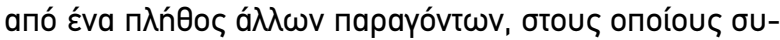

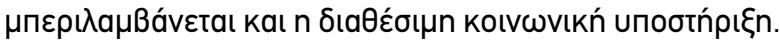

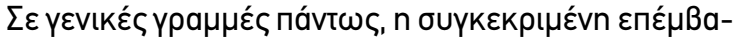

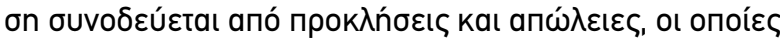

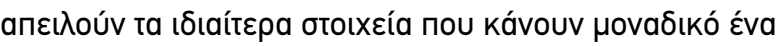

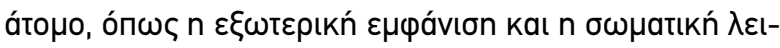

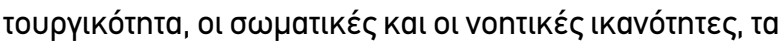

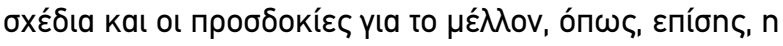

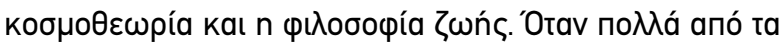

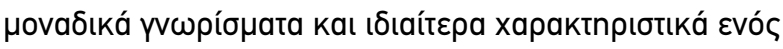

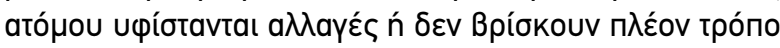

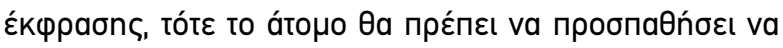

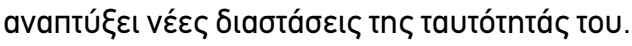

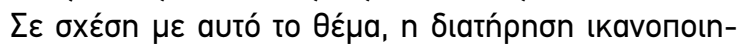

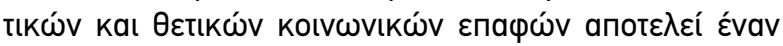

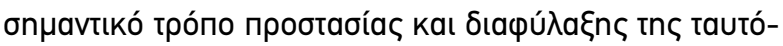

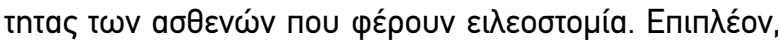

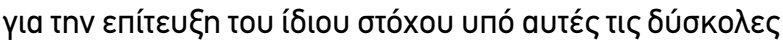

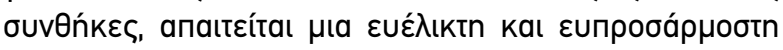

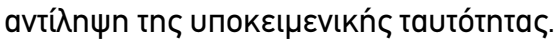

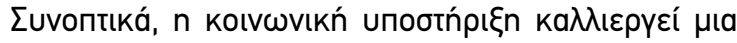

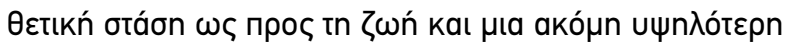

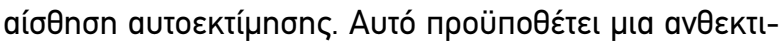

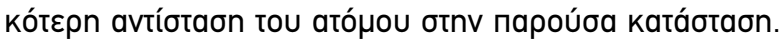

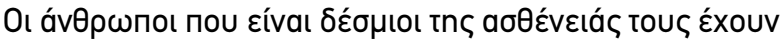

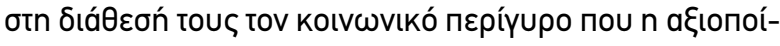

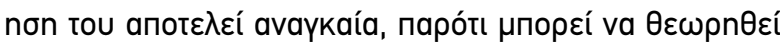

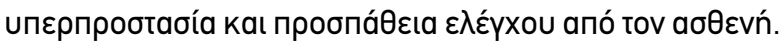

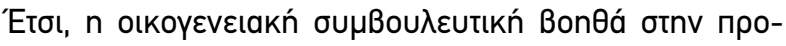

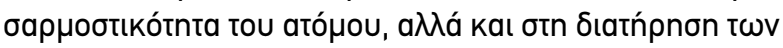

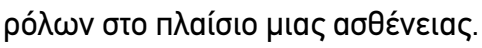

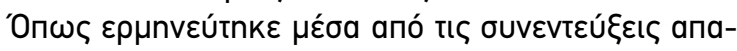

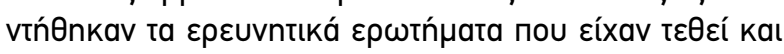

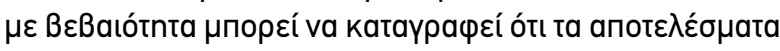

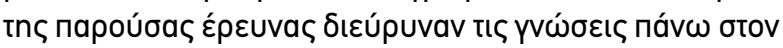

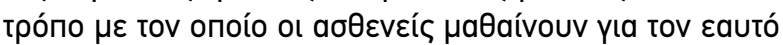

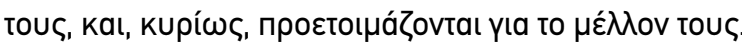

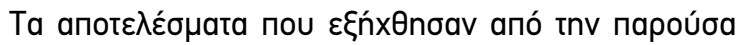

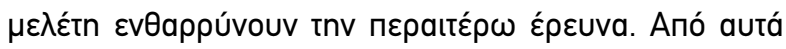




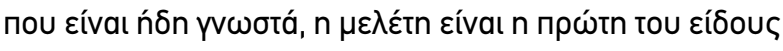

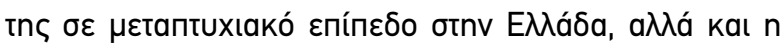

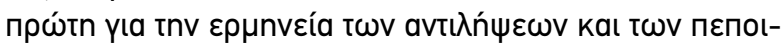

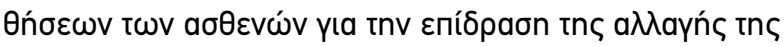

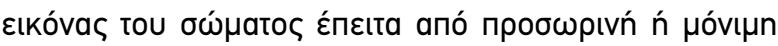
$\varepsilon 1 \lambda \varepsilon$ вотоні́́.

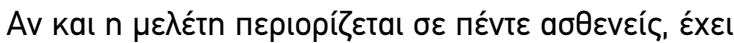

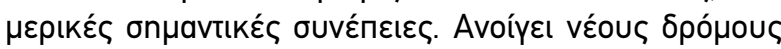

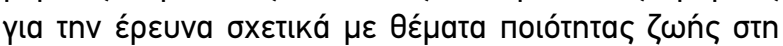

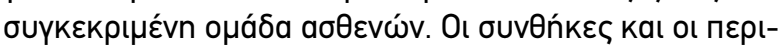

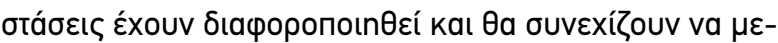

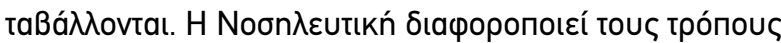

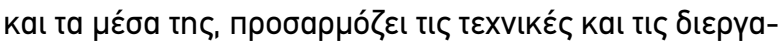

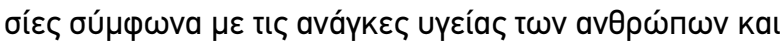

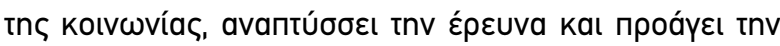

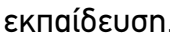

\section{Euxaplotícs}

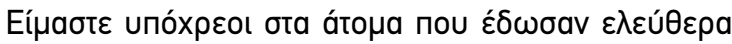

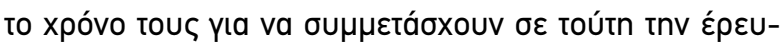

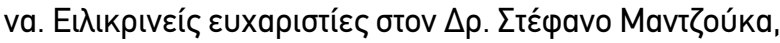

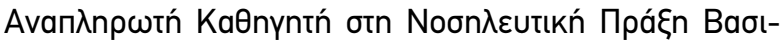

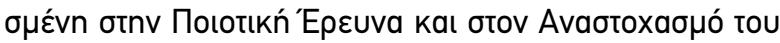

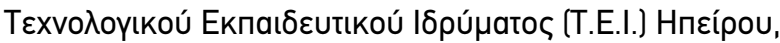

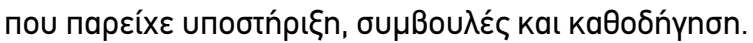

\section{ВIВАІОГРАФІА}

Alexander B., Rubinstein R., Goodman M., Luborsky M. (1992). A Path Not Taken: A Cultural Analysis of Regrets and Childlessness in the Lives of Older Women. The Gerontologist. 32(5):618-26.

Andersson G., Engstrom A., Soderberg S. (2010). A chance to live: Women's experiences of living with a colostomy after rectal cancer surgery. International Journal of Nursing Practice. 16(6): 603-608.

Backes M.T.S., Backes D.S., Erdmann A.L. (2012). Feelings and Expectations of Permanent Colostomy Patients. Journal of Nursing Education and Practice. 2(3):9-14.

Banner D.J., Albarrran J.W. (2009). "Computer-assisted qualitative data analysis software: a review". Canadian journal of cardiovascular nursing. 19 (3): 24-31.

Bossema E.R., Seuntiens W.M., Marijnen C.A., et al. (2011). The relation between illness cognitions and quality of life in people with and without a stoma following rectal cancer treatment. Psycho-Oncology. 20(4):428-434

Cascais A., Martini J.G., Almeida P.J.S. (2007). Ostomy impact in the process of human living. Texto Contexto Enferm. 16(1):163-67.

Cetolin S., Beltrame V., Cetolin S., Presta A. (2013). Social and family dynamic with patients with definitive intestinal ostomy. Arq Bras Cir Dig. 26(3):170-2

Crosby A., DiClemente R.J., Salazar L.F. (2006). Introduction to Scientific Writing Research Methods in Health promotion. John Wiley \& Sons Publications.

Dabirian A., Yaghmaei F., Rassouli M., et. al. (2011). Quality of life in ostomy patients: a qualitative study. Patient Prefer Adherence. 5: $1-5$.

Darviri C. (2009). Research methodology in the field of health. Athens, Medical Pub.: P. Ch. Paschalidis.

Duitsman D.M., \& Cychosz C.M. (1994). Phychosocial similarities and diggerences among employed and unemployed heart transplant recipients. Journal of Heart Lung Transplant. 13(1):108-15.

Dunn J., Lynch B., Rinaldis M., et al. (2006). Dimensions of quality of life and psychosocial variables most salient to colorectal cancer patients. Psycho-Oncology. 15(1):20-30

Guest G., MacQueen N. (2012). "Introduction to Thematic Analysis". Applied Thematic Analysis.

Hedman E., Ljotsson B., Lindefors N. (2012). Cognitive behavior therapy via the Internet: a systematic review of applications, clinical efficacy and cost-effectiveness. Expert Rev Pharmacoecon Outcomes Res. 12:745-64.

Hong K., Kim E., Chung S., Kim K. (2014). Psychological attitude to self-appraisal of stoma patients: prospective observation of stoma duration effect to self-appraisal. Ann Surg Treat Res. 86(3): 152-160.

Hsieh H.F., \& Shannon S. (2005). Three Approaches to Qualitative Content Analysis. Qualitative Health Research, 14(9), 1277-1288.

Kasparek M.S., Hassan I., Cima R.R., et al. (2012). Long-term Quality of Life and Sexual and Urinary Function After Abdominoperineal Resection for Distal Rectal Cancer. Dis Colon Rectum. 55(2):147154.

Krouse R.S., Grant M., Rawl S.M., et al. (2009). Coping and acceptance: the greatest challenge for veterans with intestinal stomas. $J$ Psychosom Res. 66(3):227-233.

Kyriazi N. (1999). Sociological Research. Critical Overview of Methods and Techniques. Athens: Greek Letters.

MacArthur A. (1996). Sexuality and the stoma: helping patients to cope. Nurs Times. 92:34-5.

Mancuso S., Knoesen N., Castle D.J. (2010). Delusional vs nondelusional body dysmorphic disorder. Compr Psychiatry. $51: 177-182$

Mantzoukas S. (2004). Issues of representation within qualitative inquiry. Qual Health Res, 14:994-1007.

Marques L., Weingarden H.M., Leblanc N.J., et al. (2011). Treatment utilization and barriers to treatment engagement among people with body dysmorphic symptoms. J Psychosom Res. 70:286-93.

Marquis P., Marrel A., Jambon B. (2003). Quality of life in patients with stomas: the Montreux Study. Ostomy Wound Manage; 49:48-55.

Mauricio V.C., Souza O., Lisboa M. (2014). Biopsychosocial determinants of the labor inclusion process of the person with a stoma. Rev. bras. enferm. 67(3).

Nordstrom G.M., Nyman C.R. (1991). Living with a urostomy. A follow up with special regard to the peristomal-skin complications, psychosocial and sexual life. Scand J Urol Nephrol Suppl. 138: 247-51.

Nugent K.P., Daniels P., Stewart B., et al. (1999). Quality of life in stoma patients. Dis Colon Rectum. 42:1569-74.

Ohman U. (1982). Prognosis in patients with obstructive colorectal carcinoma. Am J Surg. 143:742-747.

Papadopoulou L., Papoulia F. (2014). Psychosocial effects on quality of life of the patiemts with a colostomy. Preoperative Nursing. 3(3).

Parahoo K. (2006). Nursing research: Principles, process and issues. 2nd ed. Basingstoke, Palgrave Macmillan.

Persson E., Hellstrom A.L. (2002). Experiences of Swedish men and women 6 to 12 weeks after ostomy surgery. J Wound Ostomy 


\section{EPEYNHTIKA APOPA}

Continence Nurs. 29:103-108

Pervin A., Lawrence J.P. (2001). Personality Theories. Greek translation editor: Alexandropoulou A. - Daskalopoulou E., Athens, Tsoukitto.

Phillips K.A. (2009). Understanding Body Dysmorphic Disorder: an Essential Guide. New York, NY: Oxford University Press.

Recalla S., English K., Nazarali R., Mayo S., Miller D., Gray M. (2013). Ostomy Care and Management: A Systematic Review. J Wound Ostomy Continence Nurs. 40(5):489-500.

Reynolds F. (1997). Coping with chronic illness and disability though creative needlecraft. British journal of occupational Therapy. 60(8):352-356.

Salter M.J. (1992). What are the differences in body image between patients with a conventional stoma compared with those who have had a conventional stoma followed by a continent pouch? J Adv Nurs. 17:841-48.

Santos G., Lúcia V.C., Corrêa E.C. (2006). Quality of Life and Coping of Persons with Temporary and Permanent Stomas. Journal of Wound Ostomy \& Continence Nursing. 33(5):503-509.

Santos V.L.C.G., Paula C.A.D., Secoli S.R. (2008). Ostomy adult in São Paulo: a study on the cost of specialized equipment. Rev Esc Enferm USP. 42(2):249-55.

Scheer J., Luborsky M. (1991). The Cultural Context of Polio Biographies. Orthopedics. 14(11):1173-81.
Sharpe L., Patel D., Clarke S. (2011). The relationship between body image disturbance and distress in colorectal cancer patients with and without stomas. J Psychosom Res. 70(5):395-402.

Sinha A., Goyal H., Singh S., Rana S.P. (2009). Quality of life of ostomates with the selected factors in a selected hospital of Delhi with a view to develop guidelines for the health professionals. Indian J Palliat Care. 15:111-114.

Sprunk E., Alteneder R.R. (2000). The impact of an ostomy on sexuality. Clin J Oncol Nurs. 4:85-88.

Strauss A., Corbin J. (1998). Basics of qualitative research: Techniques and procedures for developing grounded theory. 2 nd ed. Thousand Oaks, Sage Publ.

Thomas J.R. \& Nelson J.K. (1996). Research methods in physical activity. Champaign IL: Human Kinetics.

Thompson C. (1999). Qualitative research into nursing decision making: Factors for consideration in theoretical sampling. Qual Health Res. 9:815-828.

Veale D., Anson M., Miles S., et al. (2014). Efficacy of cognitive behaviour therapy versus anxiety management for body dysmorphic disorder: a randomised controlled trial. Psychother Psychosom. 83(6):341-53.

WOCN (2016). The History of the WOCN Society. Available at: https:// www.wocn.org/page/about_us [Accessed: 10 November 2016]. 


\title{
In depth understanding of the perceptions of people with ileostomy regarding their body image
}

\author{
Stefanos Digonis \\ Nurse, MSc, Faculty of Health Sciences, University of loannina
}

\begin{abstract}
Introduction: Overall, the picture that someone has for oneself as integrated and independent man can be put to severe test because of the compulsory dependence that the ileostomy causes.

Aim: The aim of this study was to investigate in depth the opinions and expectations of patients who have undergone ileostomy, with the ultimate goal of interpreting and better understanding of human emotions in the actual environment to which they belong, highlighting the underlying impact on selfimage.

Methods: Data collection was performed by individual semi-structured interviews with open-ended questions. The sample consisted of five patients who had undergone surgery ileostomy and were selected by purposive sampling. For data analysis the qualitative methodological approach and specific initial coding «in vivo» was used, as well as thematic content analysis.

Results: Nine subcategories were created. Each of them also grouped to record six categories. Afterwards, three themes derived from these categories: a) The soul stressful situation as a specific pattern of perception of self-image, b) The preservation of identity through social support: the imaginary walls of the society towards the patient, c) The subjective feeling of lack of power and lack of attractiveness because of compulsory dependency disease. The ileostomy, permanent or temporary, except of the exhaustively physical symptoms, has serious impact on the mental and social well-being by influencing the way the person sees the change in its body image, resulting in limitations in its personal and social life.

Conclusions: The reaction of a patient to his/hers disease can be affected by many variables, including the way that the patient sees himself, the he/she behaves and thinks. Changes in physical appearance, function and body integrity are usually central to the long- or short-term experience of illness and care.
\end{abstract}

Key-words: lleostomy, illness experience, quality of life, self-image, stoma complications, phenomenology-hermeneutics. 